\title{
An Efficient and Practical System for the Catalytic Oxidation of Alcohols, Aldehydes, and $\alpha, \beta$-Unsaturated Carboxylic Acids
}

\author{
Joseph M. Grill, James W. Ogle, and Stephen A. Miller* \\ Department of Chemistry, Texas A\&M University \\ College Station, Texas 77843-3255
}

\section{Supporting Information}

Supporting information available: Detailed experimental procedures and characterization data, along with details of the kinetic studies.

\section{Table of Contents}

General Considerations and Instrumentation

Experimental Procedures

S2

Details of the Kinetic Studies.

NMR Spectra<smiles></smiles>
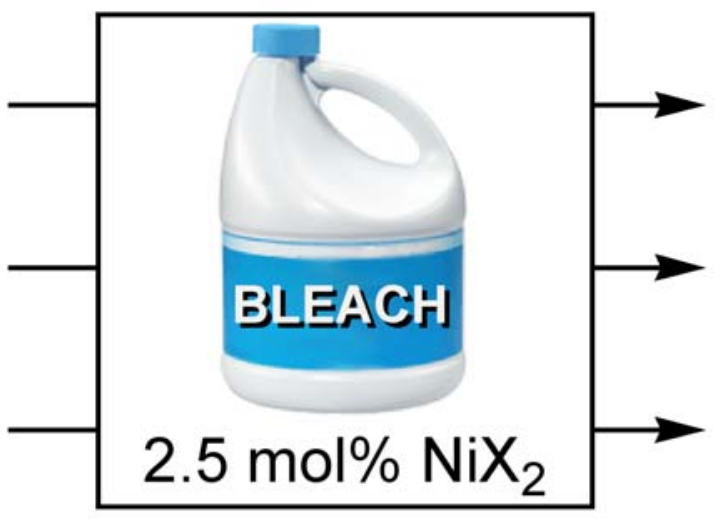<smiles>[R]C(=O)OC([R])=O</smiles> 
General Considerations and Instrumentation. Solvents were used as received. Commercial bleach ( $c a$. 5-6\% aqueous $\mathrm{NaOCl}$ ) was sparged with nitrogen for 5 minutes immediately before use. All NMR chemical shifts are given in ppm and were recorded on a Mercury-300BB or an Inova-300 spectrometer $\left({ }^{1} \mathrm{H}\right.$, $\left.299.91 \mathrm{MHz} ;{ }^{13} \mathrm{C}\left\{{ }^{1} \mathrm{H}\right\} 75.41 \mathrm{MHz}\right)$ using the solvent as an internal standard (residual proton in $\mathrm{CDCl}_{3}:{ }^{1} \mathrm{H} 7.26 \mathrm{ppm} ;{ }^{13} \mathrm{C} 77.0 \mathrm{ppm}$; or residual proton in DMSO- $\left.d_{6}:{ }^{1} \mathrm{H} 2.49 \mathrm{ppm} ;{ }^{13} \mathrm{C} 39.51 \mathrm{ppm}\right) . \mathrm{GC}$ data were obtained with an Agilent 6850 gas chromatograph with helium carrier gas and an FID detector. The column employed was a J\&W Scientific column, model 19091Z-413E with a $30 \mathrm{~m}$ length, an inner diameter of $0.32 \mathrm{~mm}$, and a film thickness of 0.25 $\mu \mathrm{m}$.

\section{Experimental Procedures}

Procedure A: Standard procedure for the oxidation of primary alcohols and aldehydes. A $500 \mathrm{~mL}$ flask was charged with $\mathrm{NiCl}_{2}$ hexahydrate $(0.27 \mathrm{~g}, 1.14 \mathrm{mmol})$ and water $(5 \mathrm{~mL})$ and allowed to dissolve. A primary alcohol or an aldehyde $(45 \mathrm{mmol})$ was added followed by dichloromethane $(15 \mathrm{~mL})$. The reaction was cooled in an ice bath after reaching homogeneity and cold bleach $(300 \mathrm{~mL})$ was added in a steady stream over 5 minutes. A fine black precipitate formed immediately. The resulting slurry was stirred for 2 hours at $0^{\circ} \mathrm{C}$ and 2 hours at room temperature. The slurry was then acidified with $2 \mathrm{M}$ hydrochloric acid until the aqueous layer was strongly acidic by $\mathrm{pH}$ paper. The aqueous layer was extracted with diethyl ether $(3 \mathrm{x} 100 \mathrm{~mL})$. The combined organic extracts were dried over anhydrous $\mathrm{MgSO}_{4}$ and filtered. Removal of the solvent by rotary evaporation and brief high vacuum gave the crude product. The purities could generally be improved to $>98 \%$ by distillation of the crude product or by recrystallization in the case of solids.

Procedure B: This procedure was identical to Procedure A except that the dichloromethane was omitted.

Procedure C: Standard procedure for the oxidation of secondary alcohols. A $500 \mathrm{~mL}$ flask was charged with $\mathrm{NiCl}_{2}$ hexahydrate $(0.27 \mathrm{~g}, 1.14 \mathrm{mmol})$ and water $(5 \mathrm{~mL})$ and allowed to dissolve. A secondary alcohol $(45 \mathrm{mmol})$ was added followed by dichloromethane $(15 \mathrm{~mL})$. The reaction was cooled in an ice bath after reaching homogeneity and cold bleach $(300 \mathrm{~mL})$ was added in a steady stream over 5 minutes. A fine black precipitate formed immediately. The resulting slurry was stirred for 2 hours at $0^{\circ} \mathrm{C}$ and 2 hours at room temperature. The aqueous layer was extracted with diethyl ether $(3 \times 100 \mathrm{~mL})$. The combined organic extracts were dried over anhydrous $\mathrm{MgSO}_{4}$ and filtered. Removal of the solvent by rotary evaporation and brief high vacuum gave the crude product. The purities could generally be improved to $>$ $98 \%$ by distillation of the crude product or by crystallization in the case of solids.

Procedure D: This procedure was identical to Procedure C except that the dichloromethane was omitted.

Procedure E: Standard procedure for the epoxidation of $\alpha, \beta$-unsaturated carboxylic acids. In a $500 \mathrm{~mL}$ round bottom flask, sodium hydroxide $(1.8 \mathrm{~g}, 45 \mathrm{mmol})$ was dissolved in water $(10 \mathrm{~mL})$ and an $\alpha, \beta$-unsaturated carboxylic acid $(45 \mathrm{mmol})$ was added, followed by dichloromethane $(15 \mathrm{~mL})$. The flask was cooled in an ice bath after reaching homogeneity and a solution of nickel(acetate $)_{2}$ tetrahydrate $(0.28 \mathrm{~g}, 1.14 \mathrm{mmol})$ in water $(5 \mathrm{~mL})$ was added. Cold bleach $(300 \mathrm{~mL})$ was added in a steady stream over 5 minutes. A fine black precipitate formed immediately. The resulting slurry was stirred for 2 hours at $0^{\circ} \mathrm{C}$ and 2 hours at room temperature. The slurry was then acidified with $2 \mathrm{M}$ hydrochloric acid until the aqueous layer was strongly acidic by $\mathrm{pH}$ paper. The aqueous layer was extracted with diethyl ether $(3 \mathrm{x}$ $100 \mathrm{~mL}$ ). The combined organic extracts were dried over anhydrous $\mathrm{MgSO}_{4}$ and filtered. Each product was then analyzed by GC.

Procedure F: This procedure was identical to Procedure E except that the dichloromethane was omitted.

Oxidation of primary alcohols. Heptanoic acid $\left(\mathrm{C}_{7} \mathrm{H}_{14} \mathrm{O}_{2}\right)$ (Table 1, entry 1). Procedure A.<smiles>CCCCCCC(=O)O</smiles>

The crude product was obtained with $80 \%$ purity by GC analysis, $5.23 \mathrm{~g} \mathrm{(89 \% ).} \mathrm{Column} \mathrm{chromatography} \mathrm{with} \mathrm{5 \%} \mathrm{ethyl}$ acetate in hexanes (Rf: 0.15 ) gave pure product by ${ }^{1} \mathrm{H}$ NMR after concentration in vacuo. Yield: $3.89 \mathrm{~g}(68 \%) .{ }^{1} \mathrm{H} \mathrm{NMR}\left(\mathrm{CDCl}_{3}\right)$ : ठ 0.80-0.96 (m, 3H), 1.19-1.34 (m, 6H), 1.54-1.68 (m, 2H), 2.34 $\left(\mathrm{t},{ }^{3} J_{\mathrm{HH}}=7.7 \mathrm{~Hz}, 2 \mathrm{H}\right), 10.72$ (broad, $\left.1 \mathrm{H}\right) .{ }^{13} \mathrm{C} \mathrm{NMR}\left(\mathrm{CDCl}_{3}\right): \delta$ 14.3, 22.7, 24.9, 28.9, 31.6, 34.3, 180.7. MS (ESI) $\mathrm{m} / z=129$ [MH]'. CAS\# [111-14-8]. Procedure B. The crude product was obtained with $92 \%$ purity by GC analysis, $5.32 \mathrm{~g}$ (91\%). Distillation gave the product with $98 \%$ purity. Yield: $5.04 \mathrm{~g}$ $(86 \%)$. The characterization data were identical to those obtained above using Procedure A.

Isobutyric acid $\left(\mathrm{C}_{4} \mathrm{H}_{8} \mathrm{O}_{2}\right)$ (Table 1, entry 2). Procedure $A$.<smiles>CC(C)C(=O)O</smiles>

The crude product was obtained with $95 \%$ purity by GC analysis, $3.76 \mathrm{~g}(95 \%)$. Distillation gave the product with $97 \%$ purity. Yield: $3.52 \mathrm{~g}(89 \%) .{ }^{1} \mathrm{H}$ NMR $\left(\mathrm{CDCl}_{3}\right): \delta 1.19\left(\mathrm{~d},{ }^{3} J_{\mathrm{HH}}=7.0 \mathrm{~Hz}\right.$, $6 \mathrm{H}), 2.58\left(\mathrm{sept},{ }^{3} J_{\mathrm{HH}}=7.0 \mathrm{~Hz}, 1 \mathrm{H}\right), 11.40$ (broad, $\left.1 \mathrm{H}\right) .{ }^{13} \mathrm{C} \mathrm{NMR}$ $\left(\mathrm{CDCl}_{3}\right): \quad \delta 19.0,34.1,184.1$. MS (ESI) $\left.\mathrm{m} / \mathrm{z}=101 \mathrm{HM}^{\mathrm{M}}\right]^{-}$. CAS\# [503-74-2]. Procedure B. The crude product was obtained with $95 \%$ purity by GC analysis, $3.81 \mathrm{~g}$ (96\%). Distillation gave the product with $97 \%$ purity. Yield: $3.53 \mathrm{~g}$ $(89 \%)$. The characterization data were identical to those obtained above using Procedure A.

Benzoic acid $\left(\mathrm{C}_{7} \mathrm{H}_{6} \mathrm{O}_{2}\right)$ (Table 1, entry 3). Procedure A.<smiles>O=C(O)c1ccccc1</smiles>

The crude product was obtained with $95 \%$ purity by GC analysis, $4.86 \mathrm{~g}(89 \%)$. The product was recrystallized from $200 \mathrm{~mL}$ of hot water. The supernatant was concentrated and the obtained material was recrystallized from $75 \mathrm{~mL}$ of water to afford a second crop. Yield: $4.28 \mathrm{~g}(78 \%) .{ }^{1} \mathrm{H} \mathrm{NMR}\left(\mathrm{CDCl}_{3}\right): \delta 7.49(\mathrm{t}$, $\left.{ }^{3} J_{\mathrm{HH}}=7.4 \mathrm{~Hz}, 2 \mathrm{H}\right), 7.63\left(\mathrm{t},{ }^{3} J_{\mathrm{HH}}=7.4 \mathrm{~Hz}, 1 \mathrm{H}\right), 8.14\left(\mathrm{~d},{ }^{3} J_{\mathrm{HH}}=7.4\right.$ $\mathrm{Hz}, 2 \mathrm{H}), 12.02$ (broad, $1 \mathrm{H}) .{ }^{13} \mathrm{C} \mathrm{NMR}\left(\mathrm{CDCl}_{3}\right): \delta 128.8,129.5$, 130.5, 134.1, 172.9. MS (ESI) $m / z=121[\mathrm{M}-\mathrm{H}]^{-}$. CAS\# [65-850]. Procedure B. The crude product was obtained with $95 \%$ purity by GC analysis, $4.77 \mathrm{~g}(87 \%)$. The product was recrystallized from ethanol. Yield: $4.10 \mathrm{~g}$ (75\%). The characterization data were identical to those obtained above using Procedure A.

Adipic acid $\left(\mathrm{C}_{6} \mathrm{H}_{10} \mathrm{O}_{4}\right)$ (Table 1, entry 4). Procedure A.<smiles>O=C(O)CCCCC(=O)O</smiles>

Note: Only $22.5 \mathrm{mmol}$ of diol were used. The product was recrystallized from water $(5 \mathrm{~mL})$. Yield: $2.24 \mathrm{~g}(68 \%)$. ${ }^{1} \mathrm{H}$ NMR $\left(\right.$ DMSO- $\left.d_{6}\right): \delta 1.47\left(\mathrm{~m},{ }^{3} J_{\mathrm{HH}}=3.0 \mathrm{~Hz}, 4 \mathrm{H}\right), 2.17\left(\mathrm{t},{ }^{3} J_{\mathrm{HH}}=3.0 \mathrm{~Hz}\right.$, 4H), 11.97 (broad, 1H). ${ }^{13} \mathrm{C}$ NMR (DMSO- $d_{6}$ ): $\delta$ 24.7, 34.0, 175.0. MS (EI) $m / z=145$ [M-H] . CAS\# [124-04-9]. Procedure B. Note: Only $22.5 \mathrm{mmol}$ of diol were used. The product was recrystallized from water. Yield: $2.37 \mathrm{~g} \mathrm{(72 \% ).} \mathrm{The}$ 
characterization data were identical to those obtained above using Procedure A.

Trimethyl acetic acid $\left(\mathrm{C}_{5} \mathrm{H}_{10} \mathrm{O}_{2}\right)$ (Table 1, entry 5). Procedure A.

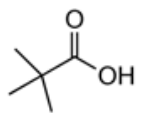

The product was obtained with $98 \%$ purity by GC analysis; thus, no further purification was necessary. Yield: $4.04 \mathrm{~g}(88 \%) .{ }^{1} \mathrm{H}$ NMR $\left(\mathrm{CDCl}_{3}\right): \delta 1.25(\mathrm{~s}, 9 \mathrm{H}), 11.09$ (broad, $\left.1 \mathrm{H}\right),{ }^{13} \mathrm{C}$ NMR $\left(\mathrm{CDCl}_{3}\right): \quad \delta$ 27.2, 38.8, 185.9. MS (ESI) $m / z=101[\mathrm{M}-\mathrm{H}]^{-}$. CAS\# [75-98-9]. Procedure B. The crude product was obtained with $97 \%$ purity by GC analysis so no further purification was required. Yield $3.72 \mathrm{~g}(81 \%)$. The characterization data were identical to those obtained above using Procedure A.

Oxidation of secondary alcohols. 2-Octanone $\left(\mathrm{C}_{8} \mathrm{H}_{16} \mathrm{O}\right)$ (Table 2, entry 1). Procedure C.

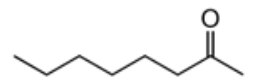

The product was obtained with $>99 \%$ purity by GC analysis; thus, no further purification was required. Yield: $4.09 \mathrm{~g}(71 \%) .{ }^{1} \mathrm{H}$ $\operatorname{NMR}\left(\mathrm{CDCl}_{3}\right): \delta 0.75\left(\mathrm{t},{ }^{3} J_{\mathrm{HH}}=6.9 \mathrm{~Hz}, 3 \mathrm{H}\right), 1.15(\mathrm{~m}, 6 \mathrm{H}), 1.43$ $(\mathrm{m}, 2 \mathrm{H}), 2.00(\mathrm{~s}, 3 \mathrm{H}), 2.29\left(\mathrm{t},{ }^{3} J_{\mathrm{HH}}=7.4 \mathrm{~Hz}, 2 \mathrm{H}\right),{ }^{13} \mathrm{C} \mathrm{NMR}$ $\left(\mathrm{CDCl}_{3}\right): \quad \delta 14.1,22.6,23.9,28.9,29.8,31.7,43.8,209.2$. MS (EI) $\mathrm{m} / z=128[\mathrm{M}+\cdot]$. CAS\# [111-13-7]. Procedure D. The product was obtained in $>99 \%$ purity by GC analysis; thus, no further purification was required. Yield: $4.04 \mathrm{~g}$, (70\%). The characterization data were identical to those obtained above using Procedure C.

Cyclopentanone $\left(\mathrm{C}_{5} \mathrm{H}_{8} \mathrm{O}\right)$ (Table 2, entry 2). Procedure $\mathrm{C}$.

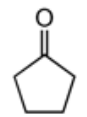

The product was obtained with $94 \%$ purity by GC analysis, $2.46 \mathrm{~g}$ $(65 \%)$. Distillation gave $>99 \%$ purity. Yield: $2.25 \mathrm{~g}(59 \%) .{ }^{1} \mathrm{H}$ NMR $\left(\mathrm{CDCl}_{3}\right): \quad \delta$ 1.71-1.82 (m, 4H), 1.91-2.00 (m, 4H). ${ }^{13} \mathrm{C}$ NMR $\left(\mathrm{CDCl}_{3}\right): \delta 23.3,38.3,220.4$. MS (EI) $\mathrm{m} / \mathrm{z}=84[\mathrm{M+} \cdot]$. CAS\# [120-92-3]. Procedure D. The product was obtained in $97 \%$ purity by GC analysis; thus, no further purification was required. Yield: $2.50 \mathrm{~g}(66 \%)$. The characterization data were identical to those obtained above using Procedure $\mathrm{C}$.

Cyclohexanone $\left(\mathrm{C}_{6} \mathrm{H}_{10} \mathrm{O}\right)$ (Table 2, entry 3). Procedure $\mathrm{C}$.<smiles>O=C1CCCCC1</smiles>

Procedure $\mathrm{C}$ was modified by allowing the reaction to stir only for 30 minutes. The product was obtained with $82 \%$ purity by GC analysis, $3.16 \mathrm{~g}(72 \%)$. Distillation gave pure product by ${ }^{1} \mathrm{H}$ NMR. Yield: $2.27 \mathrm{~g}(52 \%) .{ }^{1} \mathrm{H}$ NMR $\left(\mathrm{CDCl}_{3}\right): \delta 1.63-1.73(\mathrm{~m}$, $2 \mathrm{H}), 1.77-1.87(\mathrm{~m}, 4 \mathrm{H}), 2.30\left(\mathrm{t},{ }^{3} J_{\mathrm{HH}}=6.9 \mathrm{~Hz}, 4 \mathrm{H}\right) .{ }^{13} \mathrm{C} \mathrm{NMR}$ $\left(\mathrm{CDCl}_{3}\right): \delta$ 25.2, 27.2, 42.2, 212.5. MS (EI) $\mathrm{m} / \mathrm{z}=98[\mathrm{M+} \cdot]$. CAS\# [108-94-1]. Procedure D. The product was obtained in 92\% purity by GC analysis. Yield: $3.92 \mathrm{~g}(89 \%)$. Distillation gave the product with $98 \%$ purity. Yield: $3.31 \mathrm{~g}(75 \%)$. The characterization data were identical to those obtained above using Procedure C.

Acetophenone $\left(\mathrm{C}_{8} \mathrm{H}_{8} \mathrm{O}\right)$ (Table 2, entry 4). Procedure $\mathrm{C}$.<smiles>CCC(=O)c1ccccc1</smiles>

The product was obtained with $90 \%$ purity by GC analysis, $1.78 \mathrm{~g}$ $(33 \%)$. Distillation gave the product with $>99 \%$ purity. Yield: $1.55 \mathrm{~g}(29 \%)$. ${ }^{1} \mathrm{H}$ NMR $\left(\mathrm{CDCl}_{3}\right): \delta 2.60(\mathrm{~s}, 3 \mathrm{H}), 7.45\left(\mathrm{t},{ }^{3} J_{\mathrm{HH}}=\right.$ $7.3 \mathrm{~Hz}, 2 \mathrm{H}), 7.56\left(\mathrm{t},{ }^{3} J_{\mathrm{HH}}=7.3 \mathrm{~Hz}, 1 \mathrm{H}\right), 7.93-7.98(\mathrm{~m}, 2 \mathrm{H}) .{ }^{13} \mathrm{C}$ NMR $\left(\mathrm{CDCl}_{3}\right): \delta 28.9,128.5,128.8,133.4,137.3,198.4$. MS (EI) $\mathrm{m} / z=120[\mathrm{M}+\cdot]$. CAS\# [98-86-2]. Procedure D. Yield $2.94 \mathrm{~g}(55 \%)$. A mixture of starting material $(67 \%)$ and product $(33 \%)$ was obtained by GC analysis. This mixture was not characterized further.

Benzophenone $\left(\mathrm{C}_{13} \mathrm{H}_{10} \mathrm{O}\right)$ (Table 2, entry 5). Procedure C.<smiles>O=C(c1ccccc1)c1ccccc1</smiles>

The product was obtained with $>99 \%$ purity; thus, no further purification was required. Yield: $7.37 \mathrm{~g}(90 \%) .{ }^{1} \mathrm{H}$ NMR $\left(\mathrm{CDCl}_{3}\right): \delta 7.48\left(\mathrm{t},{ }^{3} J_{\mathrm{HH}}=7.3 \mathrm{~Hz}, 4 \mathrm{H}\right), 7.59\left(\mathrm{t},{ }^{3} J_{\mathrm{HH}}=7.3 \mathrm{~Hz}, 2 \mathrm{H}\right)$, 7.79-7.83 (m, 4H). ${ }^{13} \mathrm{C}$ NMR $\left(\mathrm{CDCl}_{3}\right): \delta 128.5,130.3,132.7$, 137.8, 197.0. MS (EI) $m / z=182$ [M+·]. CAS\# [119-61-9]. Procedure D. Only starting material was recovered from this reaction.

(+/-)-3-Methylcyclohexanone $\left(\mathrm{C}_{7} \mathrm{H}_{12} \mathrm{O}\right)$ (Table 2, entry 6). Procedure C.

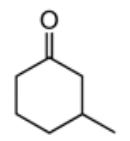

The product was obtained with $>99 \%$ purity; thus, no further purification was required. Yield: $3.93 \mathrm{~g}(78 \%) .{ }^{1} \mathrm{H}$ NMR $\left(\mathrm{CDCl}_{3}\right): \delta 0.97\left(\mathrm{~d},{ }^{3} J_{\mathrm{HH}}=6.3 \mathrm{~Hz}, 3 \mathrm{H}\right), 1.20-1.36(\mathrm{~m}, 1 \mathrm{H}), 1.53$ $1.69(\mathrm{~m}, 1 \mathrm{H}), 1.75-2.05(\mathrm{~m}, 4 \mathrm{H}), 2.12-2.36(\mathrm{~m}, 3 \mathrm{H}) .{ }^{13} \mathrm{C} \mathrm{NMR}$ $\left(\mathrm{CDCl}_{3}\right): \delta 22.3,25.5,33.5,34.4,41.3,50.2,212.2$. MS (EI) $\mathrm{m} / \mathrm{z}$ $=112[\mathrm{M+} \cdot]$. CAS\# [591-24-2]. Procedure D. Only starting material was recovered from this reaction.

2-Adamantanone $\left(\mathrm{C}_{10} \mathrm{H}_{14} \mathrm{O}\right)$ (Table 2, entry 7). Procedure C.

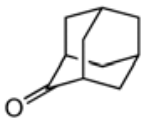

Diethyl ether was substituted for dichloromethane for this reaction because 2-adamantanol has low solubility in dichloromethane. The product was obtained with $>99 \%$ purity; thus, no further purification was required. Yield: $6.62 \mathrm{~g}(98 \%) .{ }^{1} \mathrm{H}$ NMR $\left(\mathrm{CDCl}_{3}\right): \delta 1.88-2.10(\mathrm{~m}, 12 \mathrm{H}), 2.51(\mathrm{~s}, 2 \mathrm{H}) .{ }^{13} \mathrm{C} \mathrm{NMR}\left(\mathrm{CDCl}_{3}\right)$ : $\delta$ 27.6, 36.5, 39.5, 47.2, 218.7. MS (EI) $\mathrm{m} / \mathrm{z}=150$ [M+·]. CAS\# [700-58-3]. Procedure D. Only starting material was recovered from this reaction.

(+/-)-2-tert-butylcyclohexanone $\left(\mathrm{C}_{10} \mathrm{H}_{18} \mathrm{O}\right)$ (Table 2, entry 8). Procedure C.

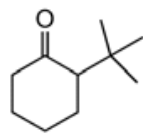

This substrate was not oxidized by the catalyst even after 24 hours

Adipic acid $\left(\mathrm{C}_{6} \mathrm{H}_{10} \mathrm{O}_{4}\right)$ (Table 2, entry 9). Procedure A.<smiles>O=C(O)CCCCC(=O)O</smiles>

Adipic acid was recovered from the oxidation of cyclohexane-1,2diol (mixture of diastereomers). Yield: $2.96 \mathrm{~g}$ (90\%). Characterization data were identical to those obtained from the 
oxidation of 1,6-hexanediol. Procedure B. Yield: $2.89 \mathrm{~g}(88 \%)$. Characterization data were identical to those obtained from the oxidation of 1,6-hexanediol.

Oxidation of aldehydes. Hexanoic acid $\left(\mathrm{C}_{6} \mathrm{H}_{12} \mathrm{O}_{2}\right)$ (Table 3, entry 1). Procedure A.

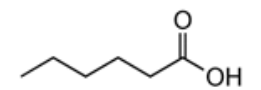

Hexanal was oxidized according to Procedure A. The product was obtained with $64 \%$ purity by GC, $4.82 \mathrm{~g}(92 \%)$. Distillation gave pure product by ${ }^{1} \mathrm{H}$ NMR. Yield $2.51 \mathrm{~g}(48 \%)$. ${ }^{1} \mathrm{H}$ NMR $\left(\mathrm{CDCl}_{3}\right): \delta$ 0.89-0.94 (m, 3H), 1.31-1.38 (m, 4H), 1.60-1.68 (m, $2 \mathrm{H}), 2.37\left(\mathrm{t},{ }^{3} \mathrm{~J}_{\mathrm{HH}}=7.8 \mathrm{~Hz}, 2 \mathrm{H}\right), 11.85$ (broad, $\left.1 \mathrm{H}\right) .{ }^{13} \mathrm{C} \mathrm{NMR}$ $\left(\mathrm{CDCl}_{3}\right): \delta 14.1,22.6,24.6,31.4,34.3,180.8$. MS (ESI) $\mathrm{m} / \mathrm{z}=$ 115 [M-H]'. CAS\# [142-62-1]. Procedure B. The product was obtained in $97 \%$ purity by GC analysis; thus, no further purification was necessary. Yield: $4.00 \mathrm{~g}(77 \%)$. The characterization data were identical to those obtained above using Procedure A.

Octanoic acid $\left(\mathrm{C}_{8} \mathrm{H}_{16} \mathrm{O}_{2}\right)$ (Table 3, entry 2). Procedure A.<smiles>CCCCCCCC(=O)O</smiles>

Octanal was oxidized according to Procedure A. The product was obtained with $94 \%$ purity by GC, $5.19 \mathrm{~g}(80 \%)$. ${ }^{1} \mathrm{H}$ NMR $\left(\mathrm{CDCl}_{3}\right): \quad \delta$ 0.84-0.92 (m, 3H), 1.20-1.39 (m, 8H), 1.54-1.68 (m, $2 \mathrm{H}), 2.34\left(\mathrm{t},{ }^{3} J_{\mathrm{HH}}=7.7 \mathrm{~Hz}, 2 \mathrm{H}\right), 11.80$ (broad, $\left.1 \mathrm{H}\right),{ }^{13} \mathrm{C} \mathrm{NMR}$ $\left(\mathrm{CDCl}_{3}\right): \quad \delta 14.3,22.8,24.9,29.1,29.2,31.9,34.3,180.4 . \quad \mathrm{MS}$ (ESI) $m / z=143[\mathrm{M}-\mathrm{H}]$. CAS\# [124-07-2]. Procedure B. The product was obtained in $98 \%$ purity by GC analysis; thus, no further purification was necessary. Yield: $5.13 \mathrm{~g}(79 \%)$. The characterization data were identical to those obtained above using Procedure A.

Benzoic acid $\left(\mathrm{C}_{7} \mathrm{H}_{6} \mathrm{O}_{2}\right)$ (Table 3, entry 3). Procedure A.<smiles>O=C(O)c1ccccc1</smiles>

Benzaldehyde was oxidized according to Procedure A. The product was obtained with $>99 \%$ purity. Yield: $5.28 \mathrm{~g}(96 \%)$. The characterization data were identical to those obtained for the oxidation of benzyl alcohol (Table 1, entry 3). Procedure B. The product was obtained in $>99 \%$ purity by GC analysis; thus, no further purification was necessary. Yield: $5.39 \mathrm{~g}(98 \%)$. The characterization data were identical to those obtained above using Procedure A.

Trimethyl acetic acid $\left(\mathrm{C}_{5} \mathrm{H}_{10} \mathrm{O}_{2}\right)$ (Table 3, entry 4). Procedure A.

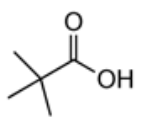

The product was obtained with $97 \%$ purity by GC analysis; thus, no further purification was necessary. Yield: $3.91 \mathrm{~g}(85 \%)$. The characterization data were identical to those obtained for the oxidation of neopentyl alcohol (Table 1, entry 5). Procedure B. The product was obtained in $98 \%$ purity by GC analysis; thus, no further purification was necessary. Yield: $4.19 \mathrm{~g}(91 \%)$. The characterization data were identical to those obtained above using Procedure A.

Epoxidation of $\boldsymbol{\alpha}, \boldsymbol{\beta}$-unsaturated carboxylic acids. All substrates in Table 4 were oxidized according to Procedure E (see reference 5) and Procedure F. The product purity was assayed by GC using standards containing pure material synthesized by an independent route ( $m$-chloroperbenzoic acid, $m$-CPBA); see below. Additional characterization data for these products can be found in reference 5 .

2-methyl-oxirane-2-carboxylic acid $\left(\mathrm{C}_{4} \mathrm{H}_{6} \mathrm{O}_{3}\right)$ (Table 4, entry 1). Procedure F.<smiles>CC1(C(=O)O)CO1</smiles>

95\% purity by GC. ${ }^{1} \mathrm{H}$ NMR $\left(\mathrm{CDCl}_{3}\right): \delta 1.58(\mathrm{~s}, 3 \mathrm{H}), 2.82(\mathrm{~d}$, $\left.{ }^{2} J_{\mathrm{HH}}=6.0 \mathrm{~Hz}, 1 \mathrm{H}\right), 3.14\left(\mathrm{~d},{ }^{2} J_{\mathrm{HH}}=6.0 \mathrm{~Hz}, 1 \mathrm{H}\right), 9.58($ broad s, $1 \mathrm{H})$. ${ }^{13} \mathrm{C} \mathrm{NMR}\left(\mathrm{CDCl}_{3}\right): \delta 17.1,53.6,53.9,176.0$. MS (ESI) $\mathrm{m} / \mathrm{z}=$ $101[\mathrm{M}-\mathrm{H}]^{-}$.

3-methyl-oxirane-2-carboxylic acid $\left(\mathrm{C}_{4} \mathrm{H}_{6} \mathrm{O}_{3}\right)$ (Table 4, entry 2). Procedure F.<smiles>CC1OC1C(=O)O</smiles>

$42 \%$ purity by GC. ${ }^{1} \mathrm{H} \mathrm{NMR}\left(\mathrm{CDCl}_{3}\right): \delta 1.45\left(\mathrm{~d},{ }^{3} J_{\mathrm{HH}}=5.1 \mathrm{~Hz}\right.$, $3 \mathrm{H}), 3.25-3.33(\mathrm{~m}, 2 \mathrm{H}), 11.10($ broad s, $1 \mathrm{H}) .{ }^{13} \mathrm{C} \mathrm{NMR}\left(\mathrm{CDCl}_{3}\right)$ : $\delta$ 17.4, 53.7, 55.4, 175.3. MS (ESI) $\mathrm{m} / \mathrm{z}=101[\mathrm{M}-\mathrm{H}]$.

3,3-dimethyl-oxirane-2-carboxylic acid $\left(\mathrm{C}_{5} \mathrm{H}_{8} \mathrm{O}_{3}\right)$ (Table 4, entry 3). Procedure F.<smiles>CC1(C)OC1C(=O)O</smiles>

$71 \%$ purity by GC. ${ }^{1} \mathrm{H}$ NMR $\left(\mathrm{CDCl}_{3}\right): \delta 1.43-1.46(\mathrm{~m}, 6 \mathrm{H}), 2.39$ (s, 1H), 9.80 (broad s, 1H). MS (ESI) $m / z=115$ [M-H] .

2,3-dimethyl-oxirane-2-carboxylic acid $\left(\mathrm{C}_{5} \mathrm{H}_{8} \mathrm{O}_{3}\right)$ (Table 4, entry 4). Procedure F.<smiles>CC1OC1(C)C(=O)O</smiles>

$85 \%$ purity by GC. ${ }^{1} \mathrm{H} \mathrm{NMR}\left(\mathrm{CDCl}_{3}\right): \delta 1.38\left(\mathrm{~d},{ }^{3} J_{\mathrm{HH}}=5.5 \mathrm{~Hz}\right.$, $3 \mathrm{H}), 1.53(\mathrm{~s}, 3 \mathrm{H}), 3.34\left(\mathrm{q},{ }^{3} J_{\mathrm{HH}}=5.5 \mathrm{~Hz}, 1 \mathrm{H}\right), 8.43$ (broad s, $\left.1 \mathrm{H}\right)$. ${ }^{13} \mathrm{C} \mathrm{NMR}\left(\mathrm{CDCl}_{3}\right): \delta 12.9,13.6,58.0,58.9,175.4$. MS (ESI) $\mathrm{m} / \mathrm{z}$ $=115[\mathrm{M}-\mathrm{H}]$.

2-methyl-oxirane-2-carboxylic acid $\left(\mathrm{C}_{4} \mathrm{H}_{6} \mathrm{O}_{3}\right)$ (Table 4, entry 5). Procedure F.<smiles>CC1(C(=O)O)CO1</smiles>

$89 \%$ purity by GC. The characterization data were identical to those obtained for the product of Table 4, entry 1.

Synthesis of epoxide standards using $\boldsymbol{m}$-chloroperbenzoic acid $\left(\boldsymbol{m}\right.$-CPBA). ${ }^{1}$ The four unique products shown in Table 4 were prepared independently to obtain "authentic samples" for NMR and GC standards. An olefin (10.0 mmol, 1.0 eq.) was dissolved in dichloromethane $(25 \mathrm{~mL})$ in a flask. Solid $m$-CPBA (77\% maximum peroxide content, $6.0 \mathrm{~g}, 26.8 \mathrm{mmol}, 2.68 \mathrm{eq}$.) was added and the reaction was stirred for 24 hours at reflux. The solution was cooled to room temperature and any solid that formed was filtered off and discarded. The dichloromethane was then removed by rotary evaporation and the resulting residue was rinsed with cold water. The slurry was filtered and the solid was discarded. The water was removed by rotary evaporation and the resulting residue was distilled or sublimed into a liquid-nitrogen cooled trap under high vacuum. ${ }^{13} \mathrm{C}$ NMR, ${ }^{1} \mathrm{H}$ NMR, and GC standards were prepared from these materials.

Oxidation of 3-butenoic acid. Procedure E.<smiles>C=CCC(=O)OCC(=O)O</smiles> 
3-Butenoic acid (3.87 $\mathrm{g}, 45 \mathrm{mmol})$ was oxidized according to Procedure E. Fumaric acid was recovered as the product. Yield $3.92 \mathrm{~g}(75 \%) . \quad{ }^{1} \mathrm{H}$ NMR (DMSO- $\left.d_{6}\right): \quad \delta 6.58$ (s, 2H), 13.08 (broad, $2 \mathrm{H}$ ). ${ }^{13} \mathrm{C}$ NMR (DMSO- $\left.d_{6}\right): \delta 134.60,166.67 . \mathrm{MS}$ (ESI) $m / z=115[\mathrm{M}-\mathrm{H}]^{-}$. CAS\# [110-17-8].

Oxidation of acrylic acid. Procedure E.<smiles>C=CC(=O)OCCCC(=O)O[OH+]</smiles>

The product was analyzed by ${ }^{1} \mathrm{H}$ NMR $(\delta 4.15 \mathrm{ppm}$ for monochloroacetic acid and $\delta 5.95 \mathrm{ppm}$ for dichloroacetic acid) and GC. The product contained a mixture of monochloroacetic acid and dichloroacetic acid in ratios that varied from 1:1 to 5:1 under seemingly identical reaction conditions. MS (ESI) $\mathrm{m} / \mathrm{z}=93$ $[\mathrm{M}-\mathrm{H}]^{-}$. MS (ESI) $m / z=127[\mathrm{M}-\mathrm{H}]^{-}$.

Oxiranylacetic acid.

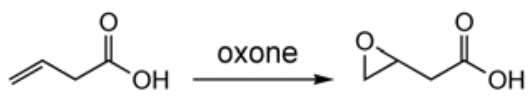

3-butenoic acid (3.00 g, $0.035 \mathrm{~mol})$ was dissolved in water (50 $\mathrm{mL})$ and acetone $(25 \mathrm{~mL})$. Sodium bicarbonate $(20.00 \mathrm{~g})$ was added. Oxone (40.00 g, $0.065 \mathrm{~mol}$ ) was added slowly via solid addition funnel. The reaction was stirred at room temperature for 4 hours. The solid was filtered off and dichloromethane $(30 \mathrm{~mL})$ was added. The aqueous layer was acidified with hydrochloric acid and then the organic layer was collected. The aqueous layer was extracted with diethyl ether $(3 \times 50 \mathrm{~mL})$. The combined organic extracts were dried over anhydrous $\mathrm{MgSO}_{4}$ and the solvent was removed by rotary evaporation. The crude product contained a $12 \%$ impurity (by GC) of 3-methyl-oxirane-2carboxylic acid due to an isomerization reaction prior to epoxidation. The mixture was used without further purification for the next step. Yield: $2.51 \mathrm{~g}(70 \%) .{ }^{1} \mathrm{H} \mathrm{NMR}\left(\mathrm{CDCl}_{3}\right): \delta$ 2.57-2.64 (m, 3H), 2.87 (m, 1H), 3.25-3.33 (m, 1H), 10.15 (broad, $1 \mathrm{H}) .{ }^{13} \mathrm{C} \mathrm{NMR}\left(\mathrm{CDCl}_{3}\right): \delta 38.0,47.0,48.0,176.8$. MS (ESI) $\mathrm{m} / \mathrm{z}$ $=101\left[^{[\mathrm{M}-\mathrm{H}}\right]^{-}$. CAS\# [33278-09-0].

Oxidation of oxiranylacetic acid. Procedure E. Note that only $1.00 \mathrm{~g}$ of oxiranylacetic acid was used. Fumaric acid was recovered as the product with a small contamination of 3-methyloxirane-2-carboxylic acid. This impurity could easily be removed by crystallization of the product from water. Yield: $0.79 \mathrm{~g}$ $(69 \%)$. Characterization data were identical to those obtained for the product of direct oxidation of 3-butenoic acid using Procedure E.

1) Brimeyer, M. O.; Mehrota, A.; Quici, S.; Nigam, A.; Regen, S. L. J. Org. Chem. 1980, 45, 4254-4255. 


\section{Details of the Kinetic Studies}

Kinetic data were obtained by using procedure $\mathrm{B}$; however, instead of cooling the reaction to $0^{\circ} \mathrm{C}$ initially, the temperature was kept constant at $20 \pm 1{ }^{\circ} \mathrm{C}$ using an external water bath. At each 5-minute time interval, a 2 mL aliquot was removed and combined with a $2 \mathrm{~mL}$ saturated aqueous sodium thiosulphate solution. This quenched the reaction $(\mathrm{NaOCl})$ immediately. After cooling, 1 drop of concentrated hydrochloric acid was added, followed by $5 \mathrm{~mL}$ of diethyl ether. A sample from the ether layer was then assayed by gas chromatography (GC). The GC was standardized by injecting a 1:1:1 molar ratio of alcohol, aldehyde, and carboxylic acid in diethyl ether. The average detector response of 3 trials was determined and applied to obtain the following data.

\begin{tabular}{cccc}
\hline time (minutes) & benzyl alcohol & benzaldehyde & benzoic acid \\
\hline 0 & 100.0 & 0.0 & 0.0 \\
5 & 94.1 & 5.9 & 0.0 \\
10 & 88.5 & 11.5 & 0.0 \\
15 & 84.4 & 14.4 & 1.2 \\
20 & 79.1 & 18.9 & 2.0 \\
25 & 73.3 & 20.4 & 6.3 \\
30 & 66.2 & 27.2 & 6.6 \\
35 & 56.3 & 28.6 & 15.2 \\
40 & 23.9 & 54.2 & 21.9 \\
45 & 8.1 & 54.3 & 37.6 \\
50 & 2.5 & 34.4 & 63.1 \\
55 & 0.5 & 8.7 & 90.8 \\
60 & 0.0 & 2.2 & 97.8 \\
65 & 0.0 & 0.2 & 99.8 \\
70 & 0.0 & 0.0 & 100.0 \\
\hline
\end{tabular}

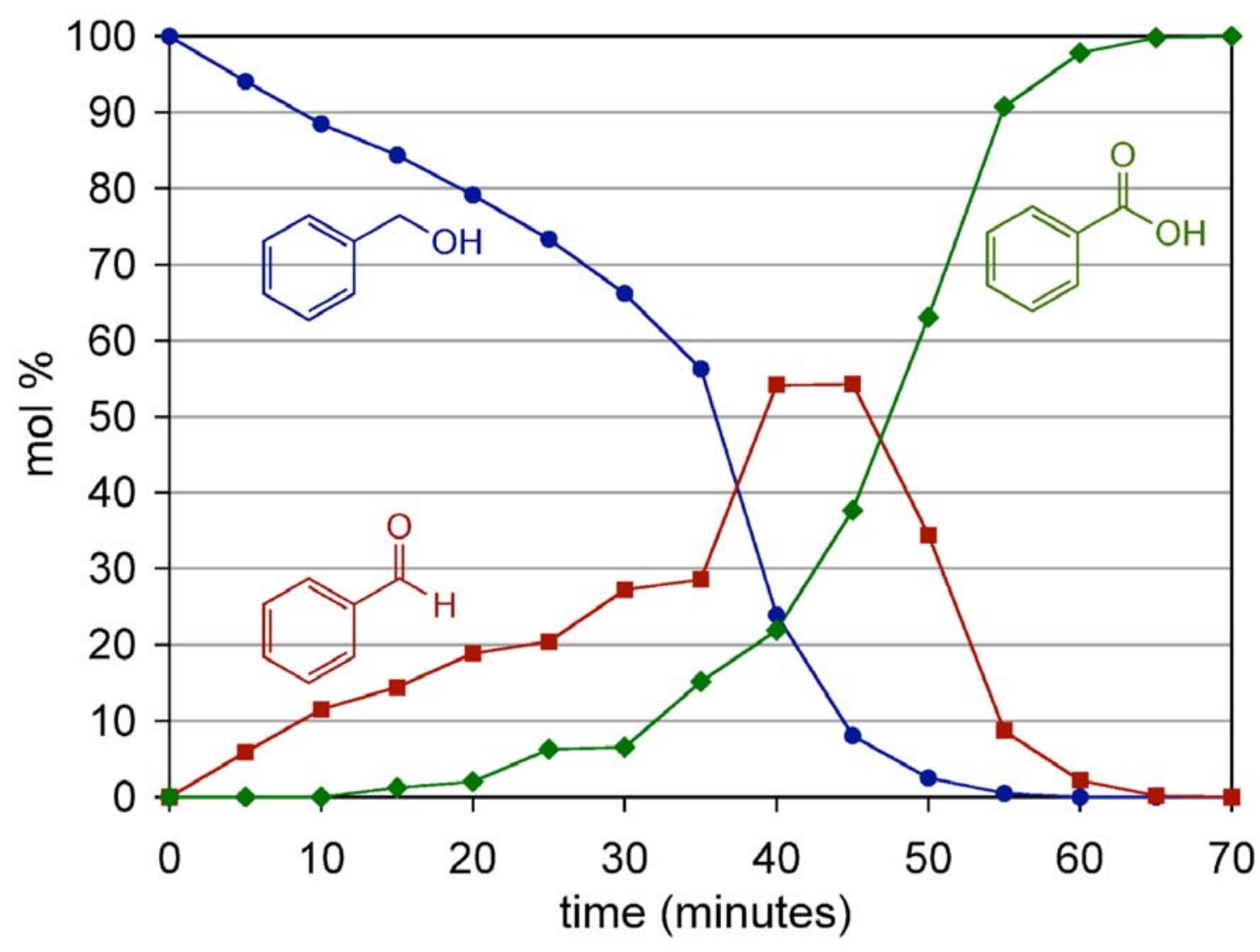




\begin{tabular}{ccc}
\hline time (minutes) & benzaldehyde & benzoic acid \\
\hline 0 & 100.0 & 0.0 \\
5 & 73.5 & 26.5 \\
10 & 43.1 & 56.9 \\
15 & 6.4 & 93.6 \\
20 & 0.1 & 99.9 \\
25 & 0.0 & 100.0 \\
30 & 0.0 & 100.0 \\
\hline
\end{tabular}

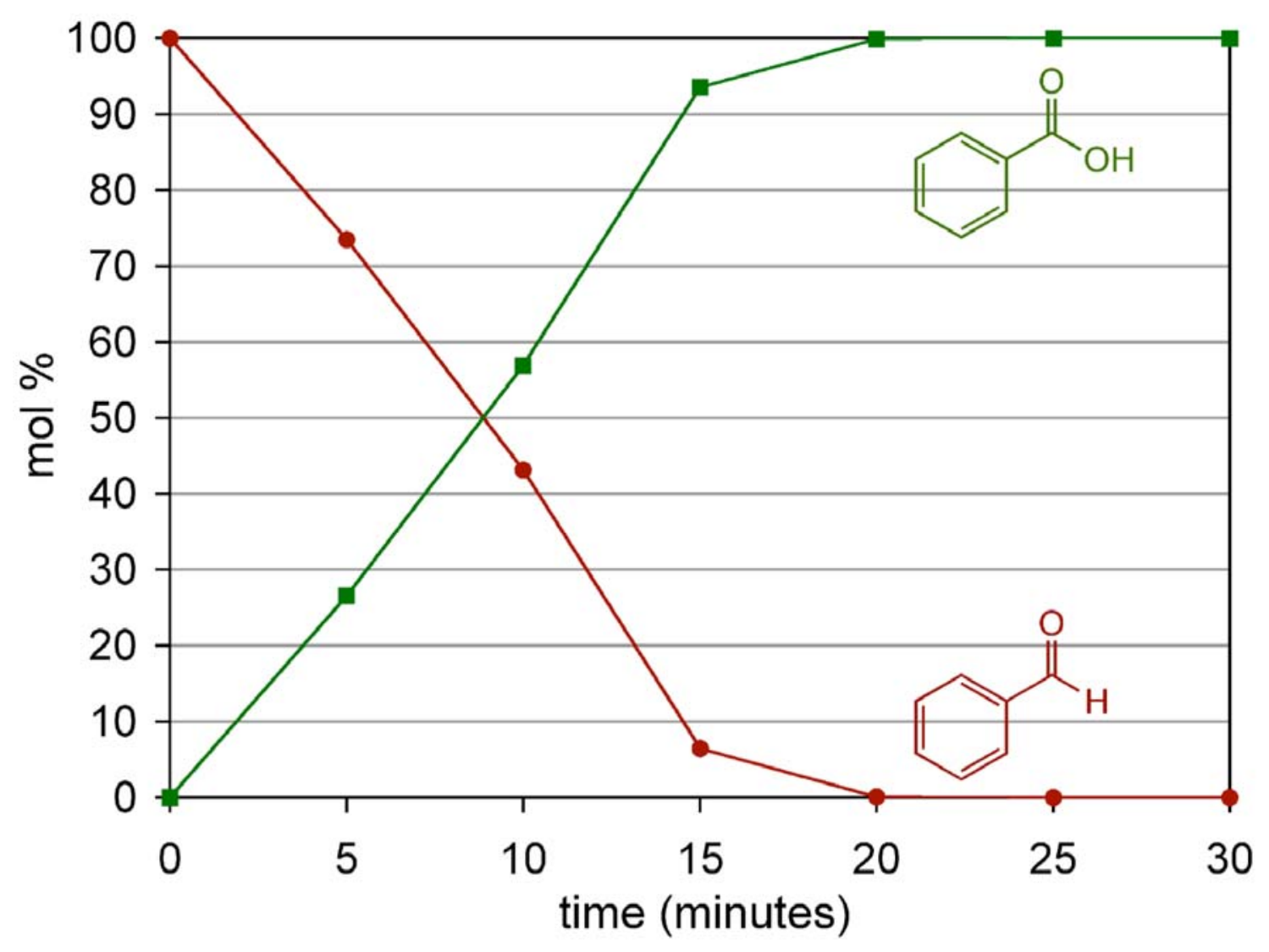




\begin{tabular}{ccc}
\hline time (minutes) & 1-propanol & propionic acid \\
\hline 0 & 100.0 & 0.0 \\
5 & 74.1 & 25.9 \\
10 & 54.3 & 45.7 \\
15 & 0.0 & 100.0 \\
20 & 0.0 & 100.0 \\
\hline
\end{tabular}

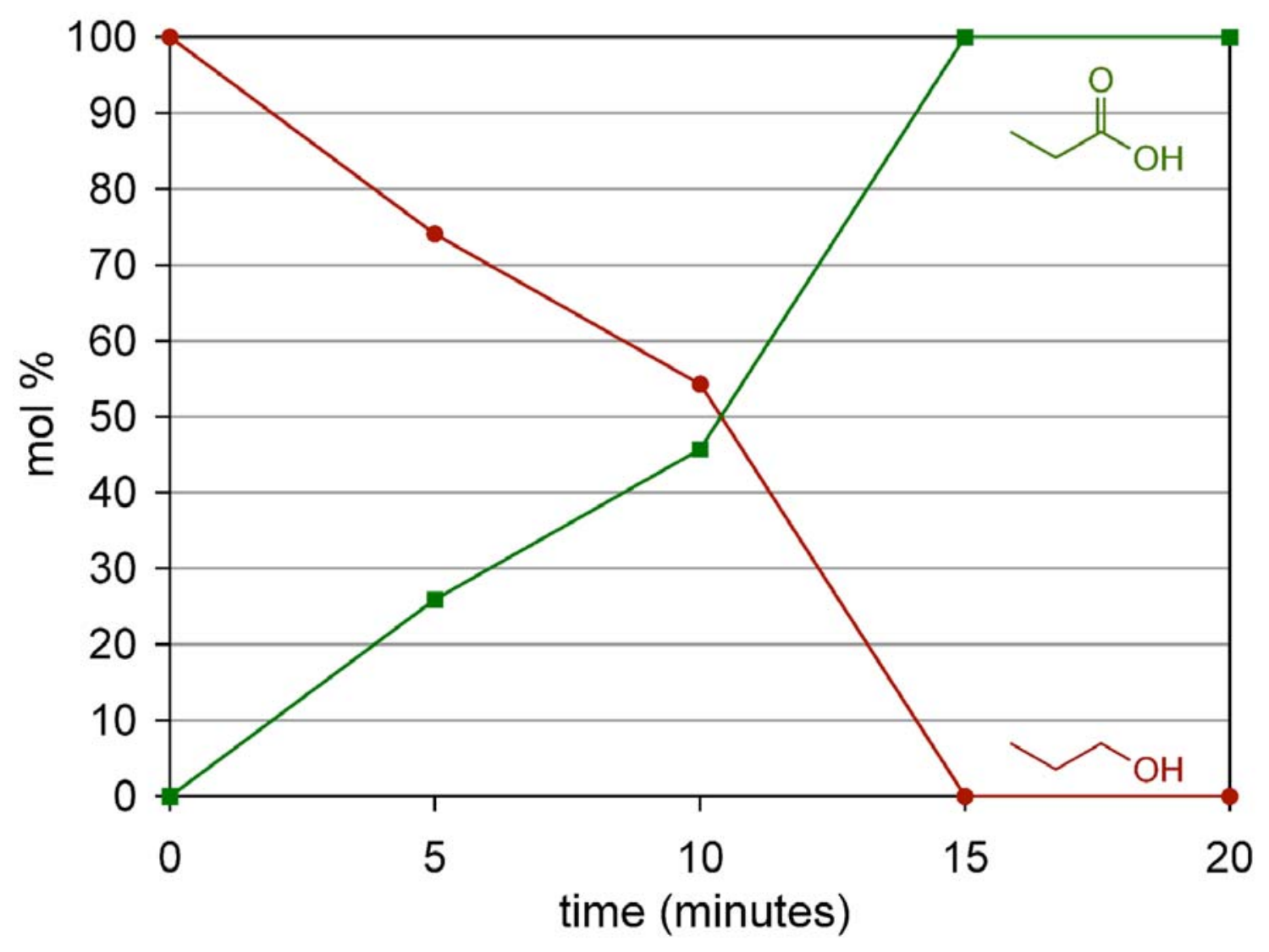




\section{NMR Spectra}

${ }^{1} \mathrm{H}$ NMR spectrum of heptanoic acid. Table 1, entry 1.
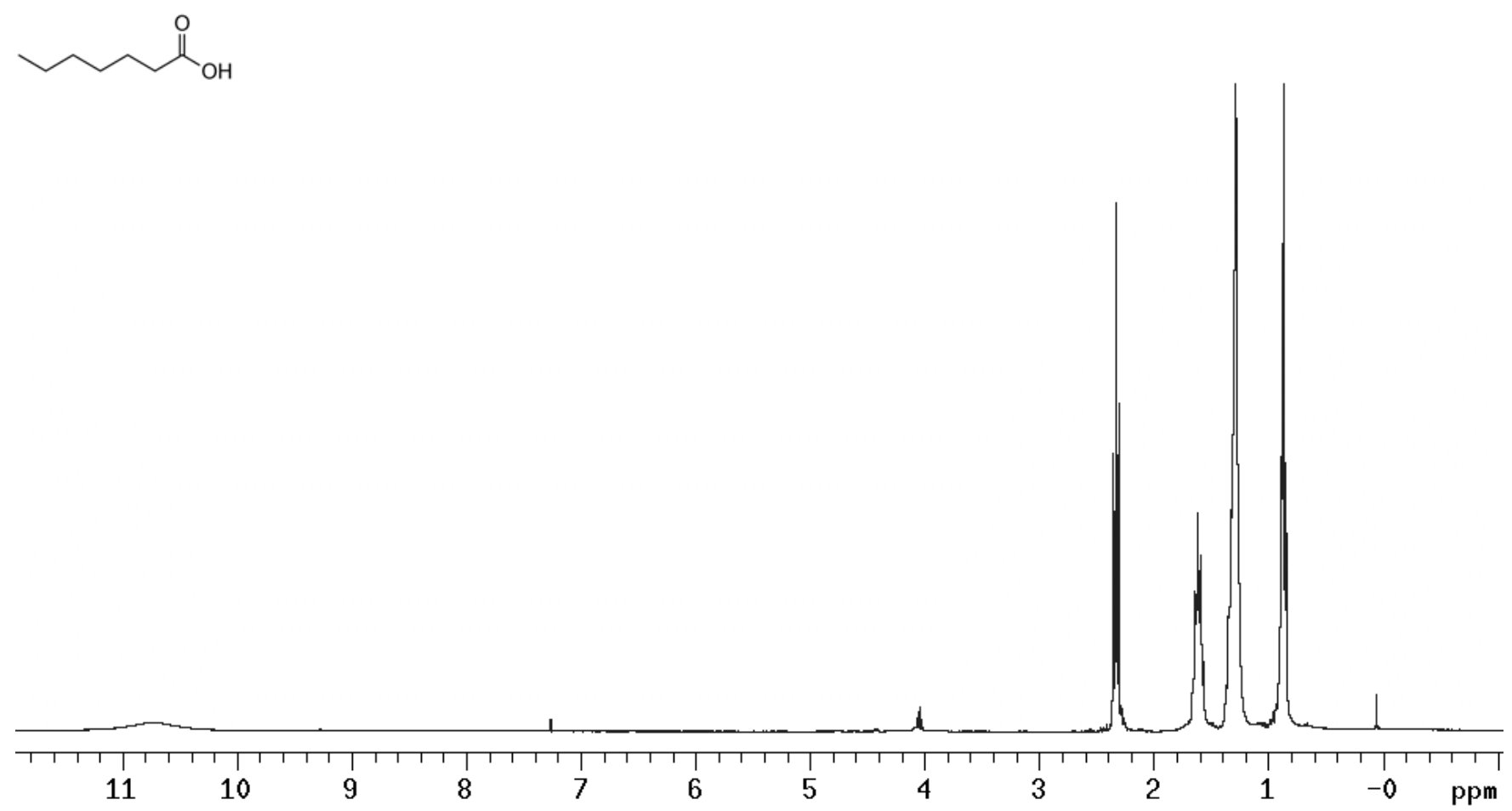

${ }^{13} \mathrm{C}$ NMR spectrum of heptanoic acid. Table 1 , entry 1.
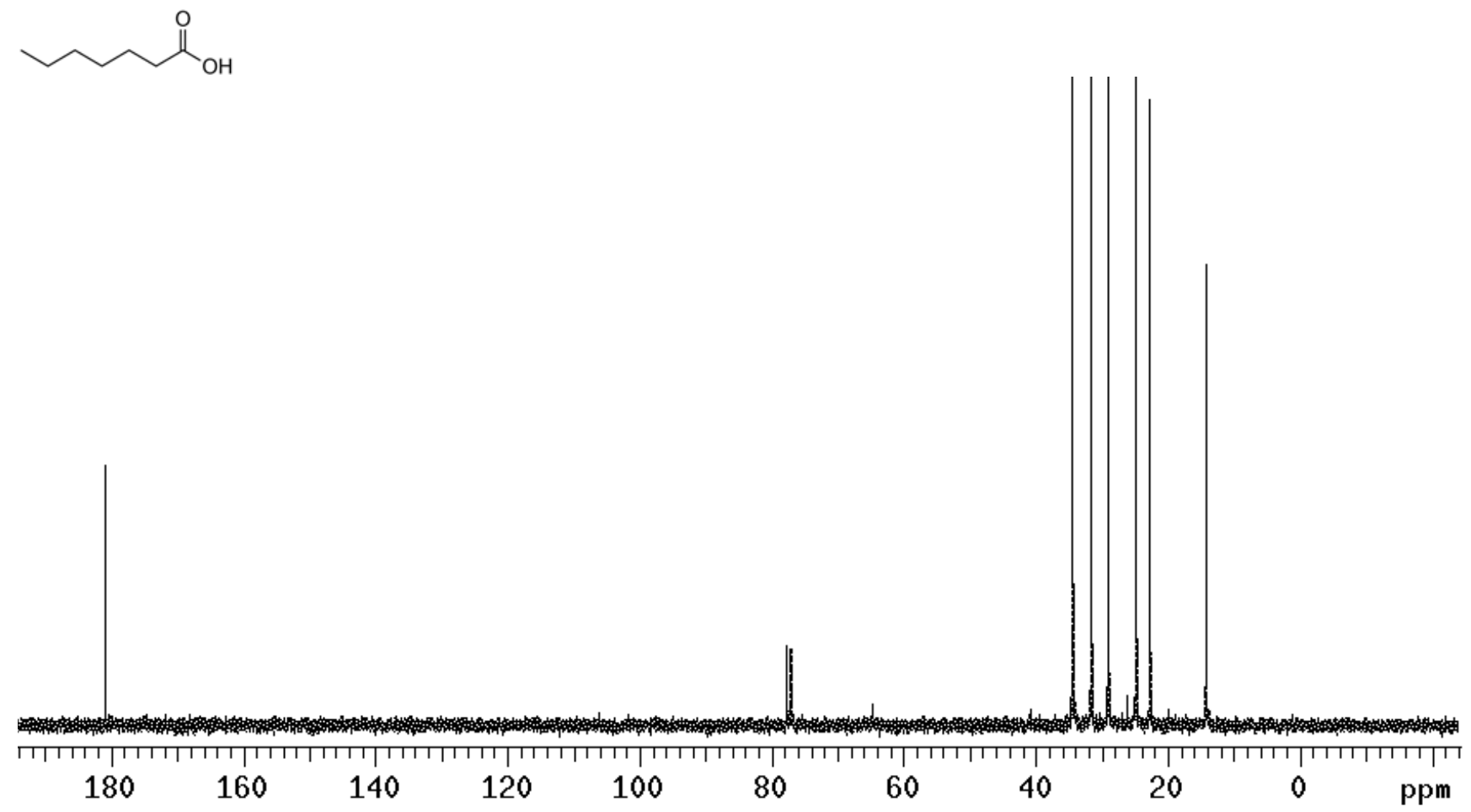
${ }^{1} \mathrm{H}$ NMR spectrum of isobutyric acid. Table 1, entry 2.<smiles>CC(C)C(=O)O</smiles>

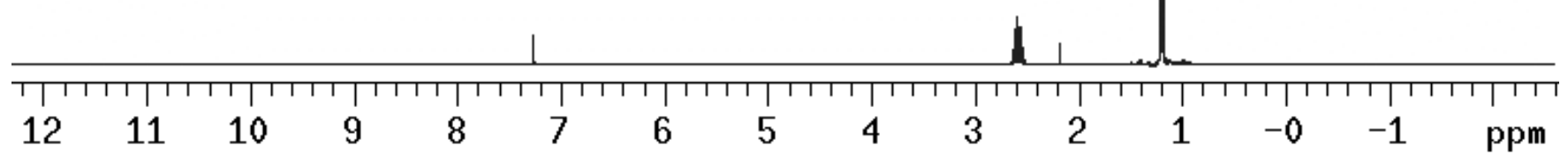

${ }^{13} \mathrm{C}$ NMR spectrum of isobutyric acid. Table 1, entry 2.
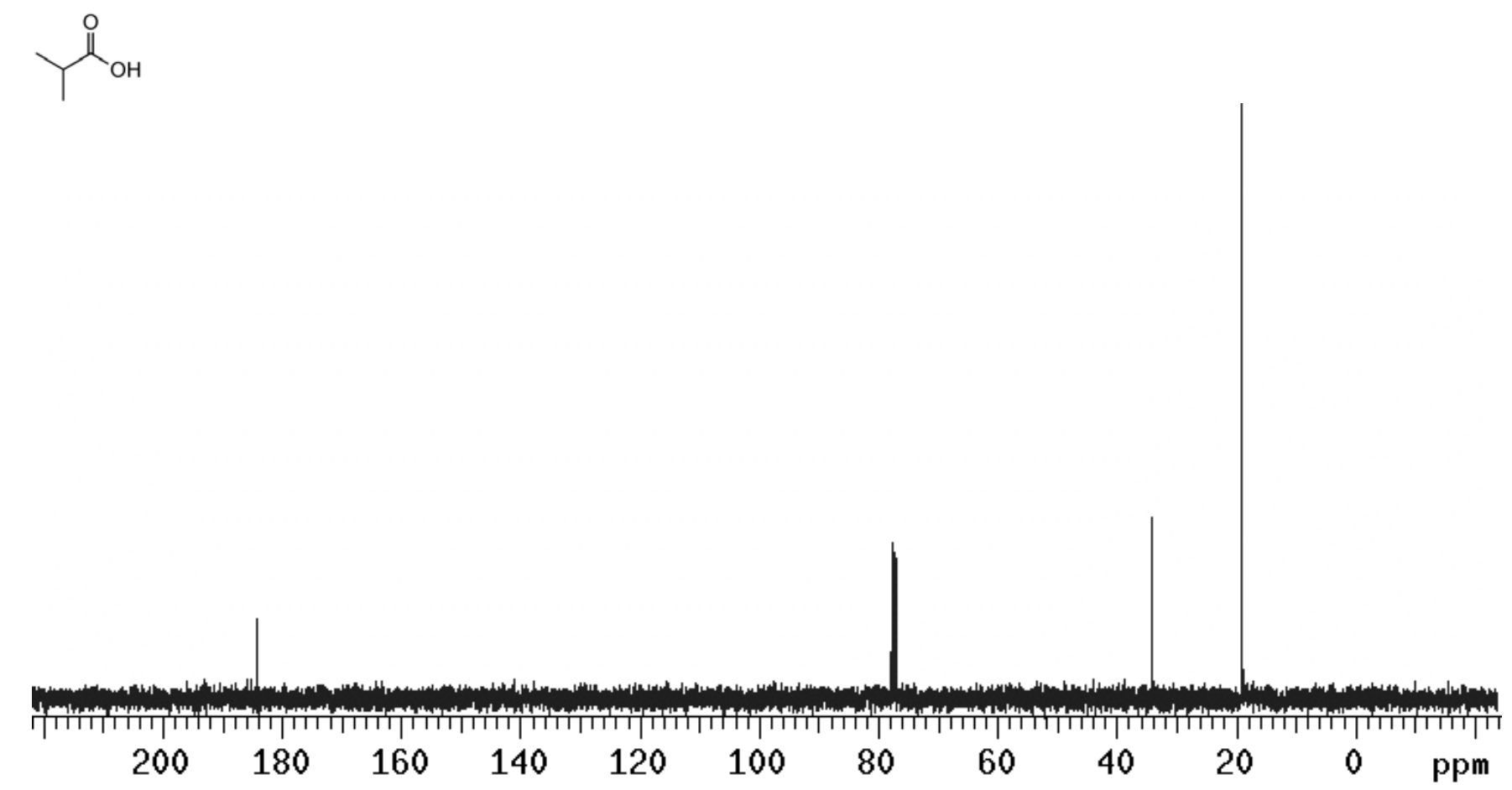
${ }^{1} \mathrm{H}$ NMR spectrum of benzoic acid. Table 1, entry 3.<smiles>O=C(O)c1ccccc1</smiles>

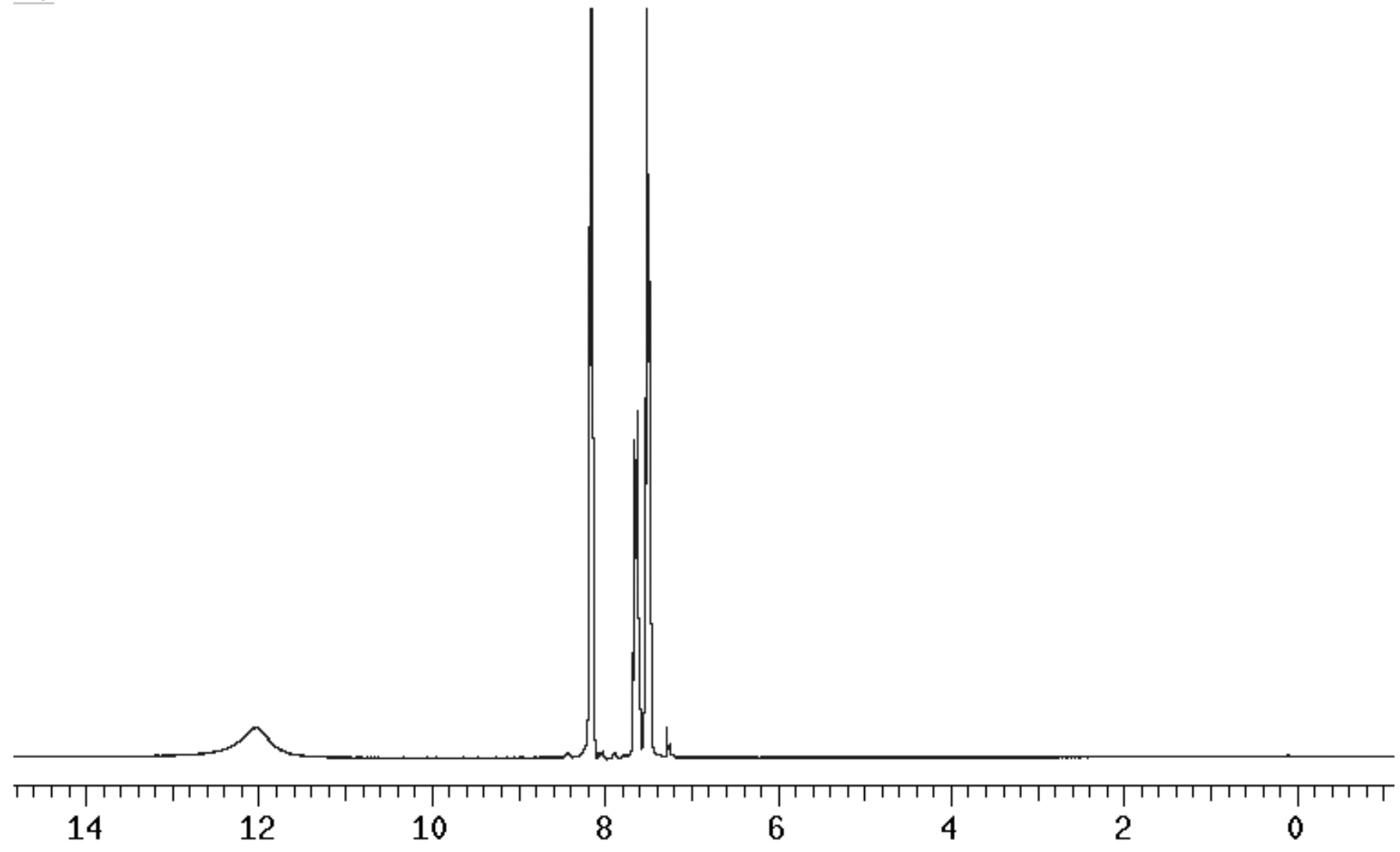

${ }^{13} \mathrm{C}$ NMR spectrum of benzoic acid. Table 1 , entry 3.<smiles>O=C(O)c1ccccc1</smiles>

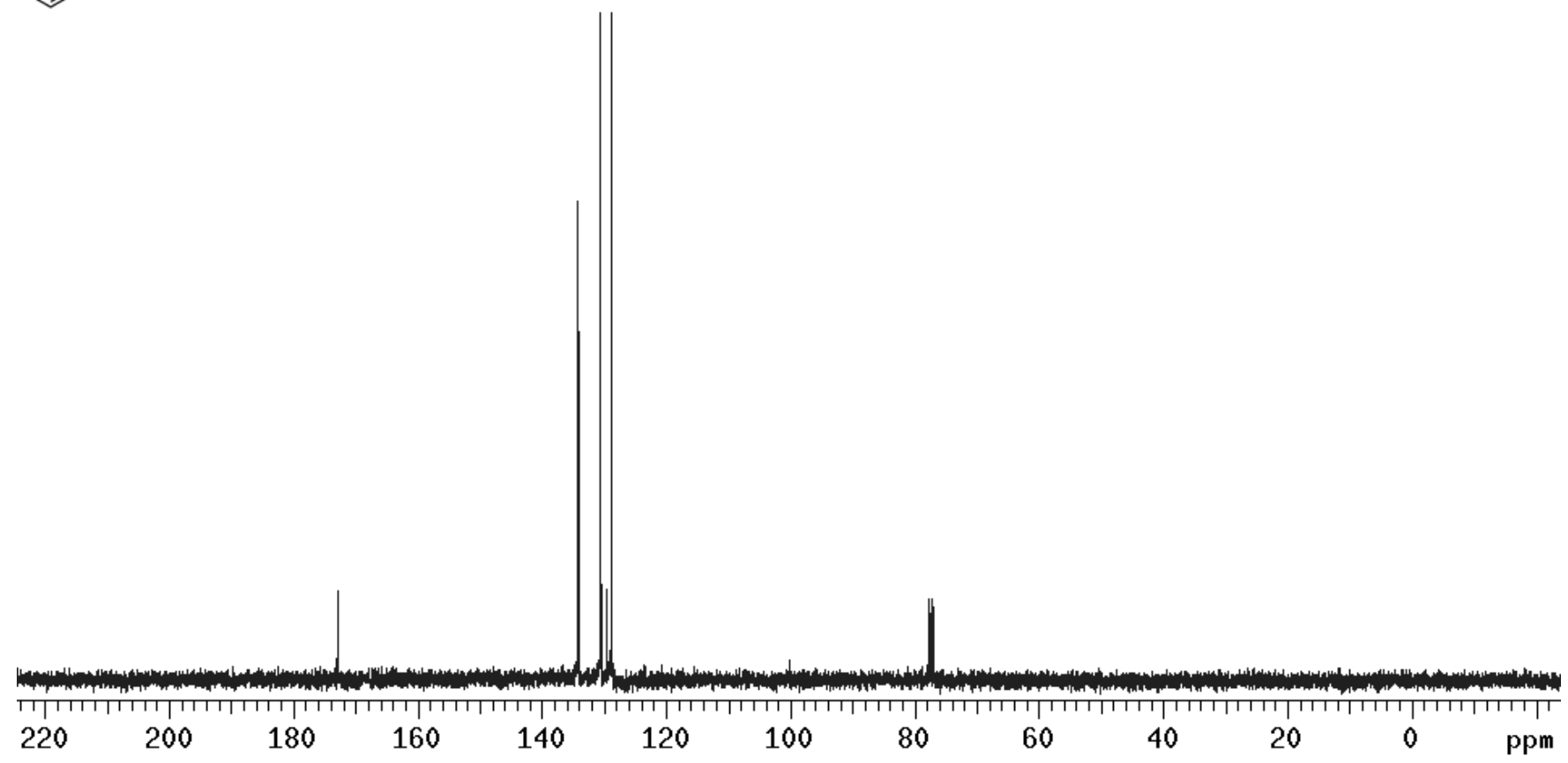

S11 
${ }^{1} \mathrm{H}$ NMR spectrum of adipic acid. Table 1, entry 4 .<smiles>O=C(O)CCCCC(=O)O</smiles>

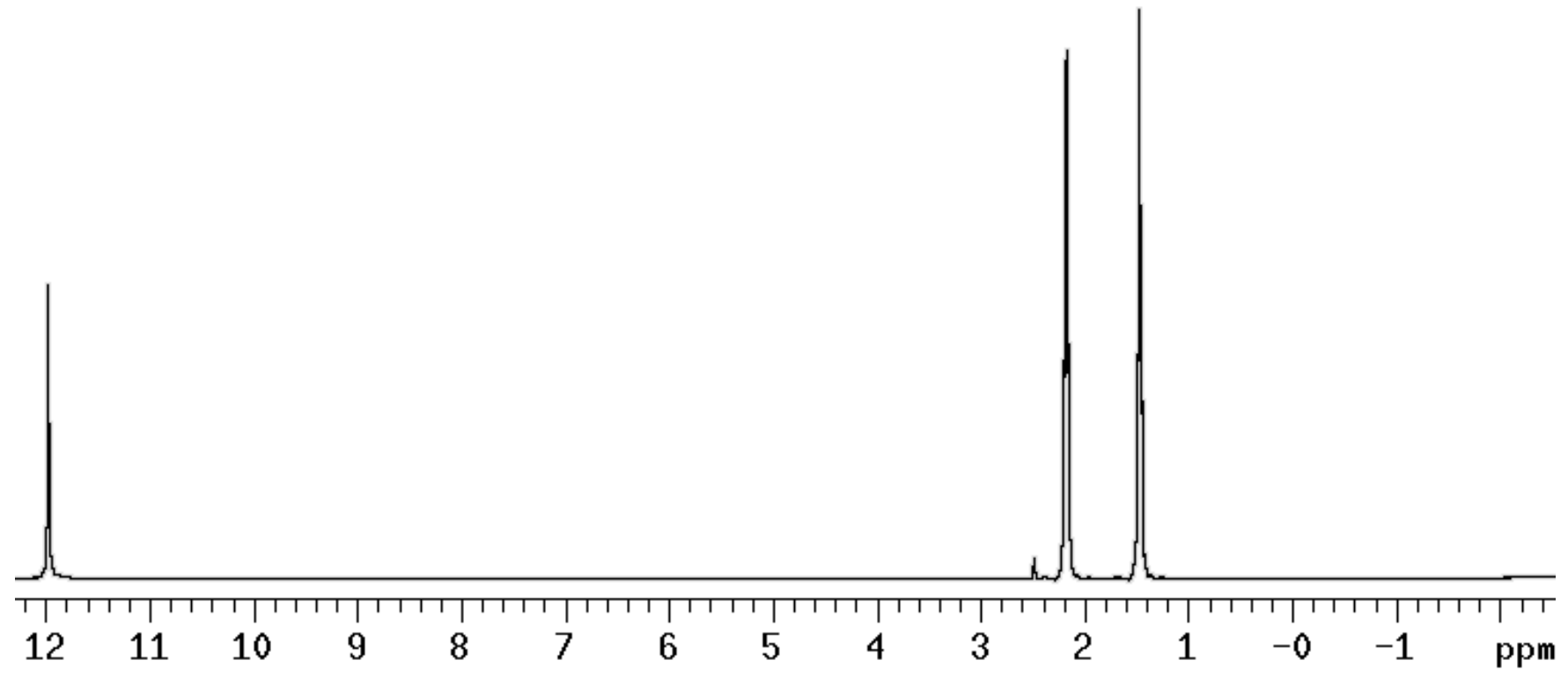

${ }^{13} \mathrm{C}$ NMR spectrum of adipic acid. Table 1, entry 4.<smiles>O=C(O)CCCCC(=O)O</smiles>

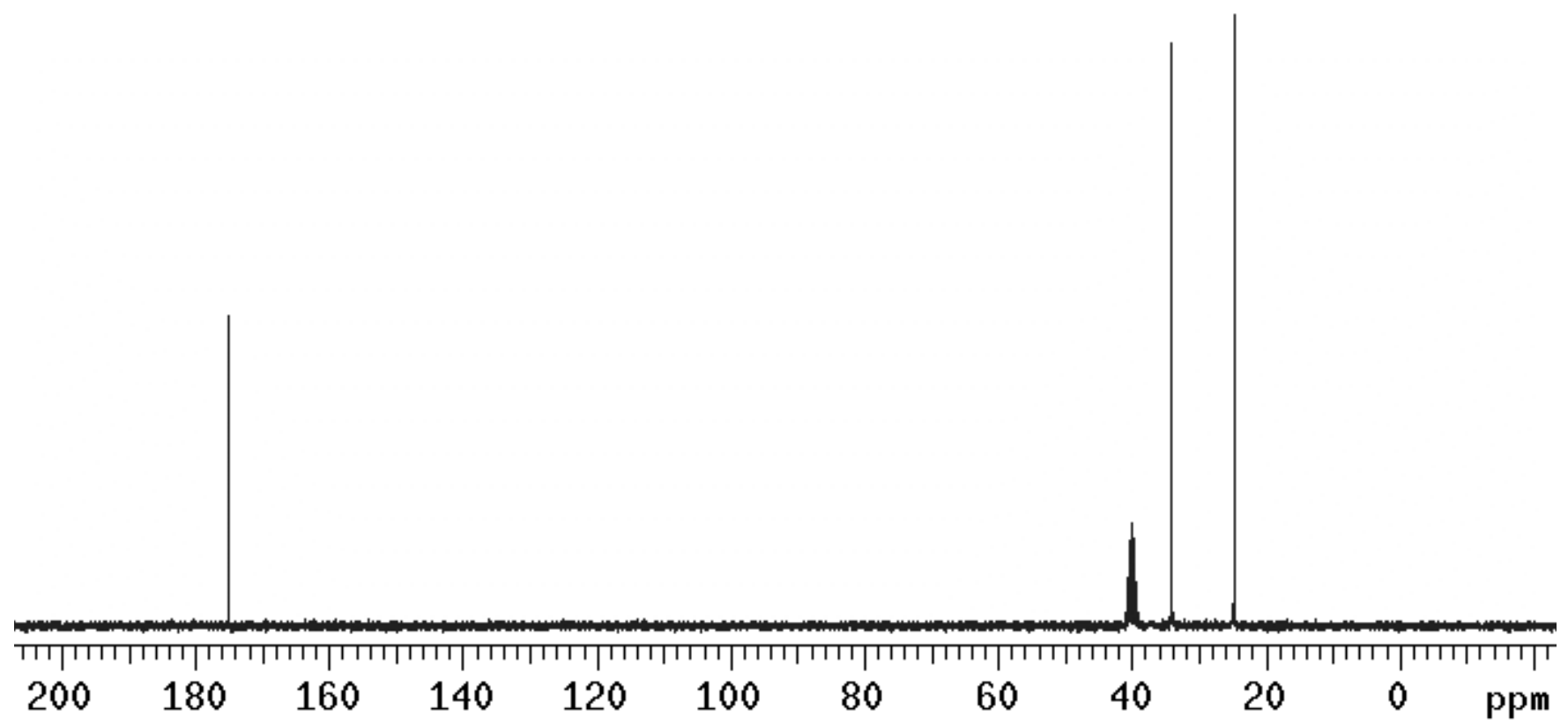


${ }^{1} \mathrm{H}$ NMR spectrum of trimethylacetic acid. Table 1, entry 5.
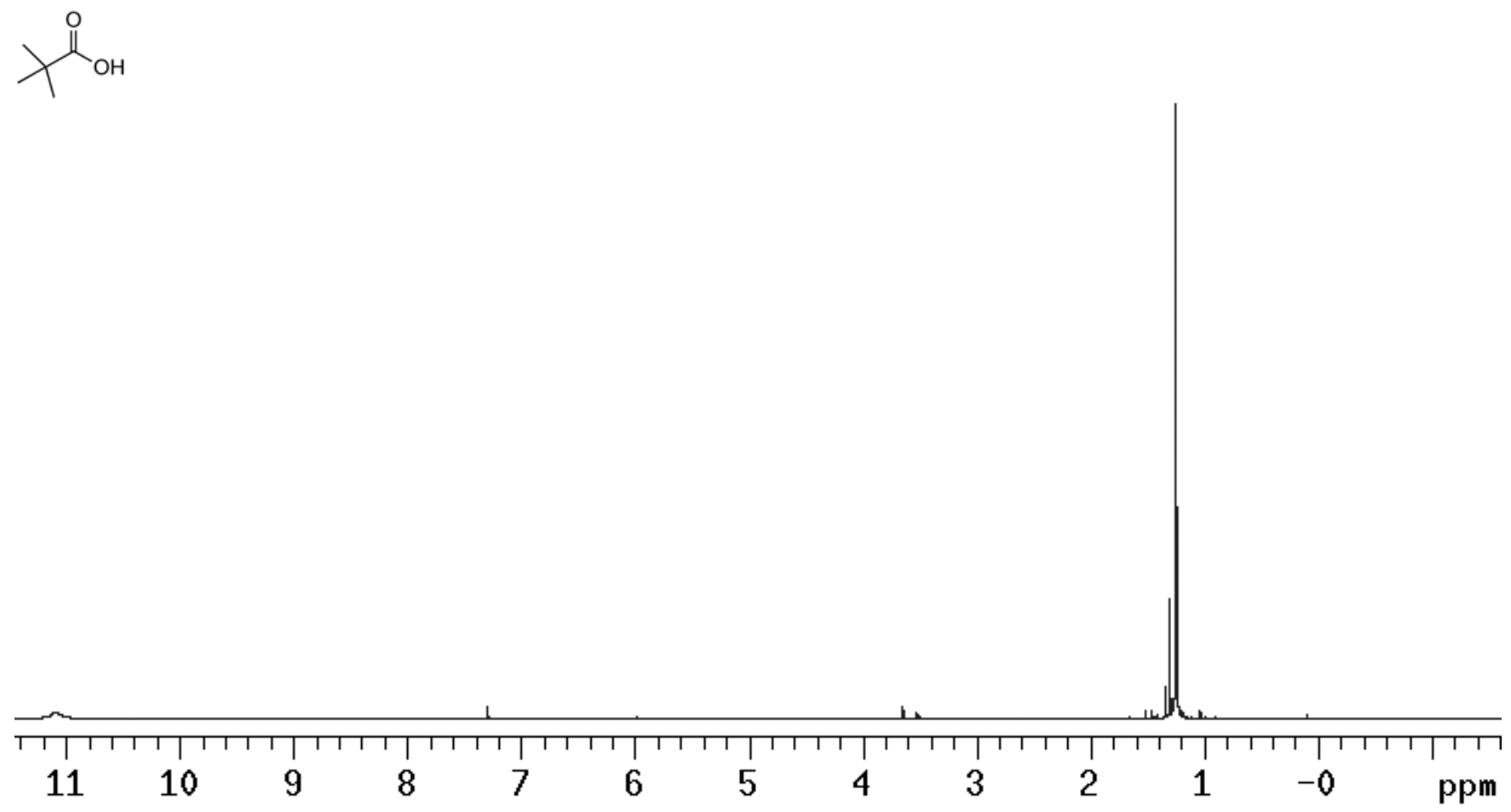

${ }^{13} \mathrm{C}$ NMR spectrum of trimethylacetic acid. Table 1 , entry 6.
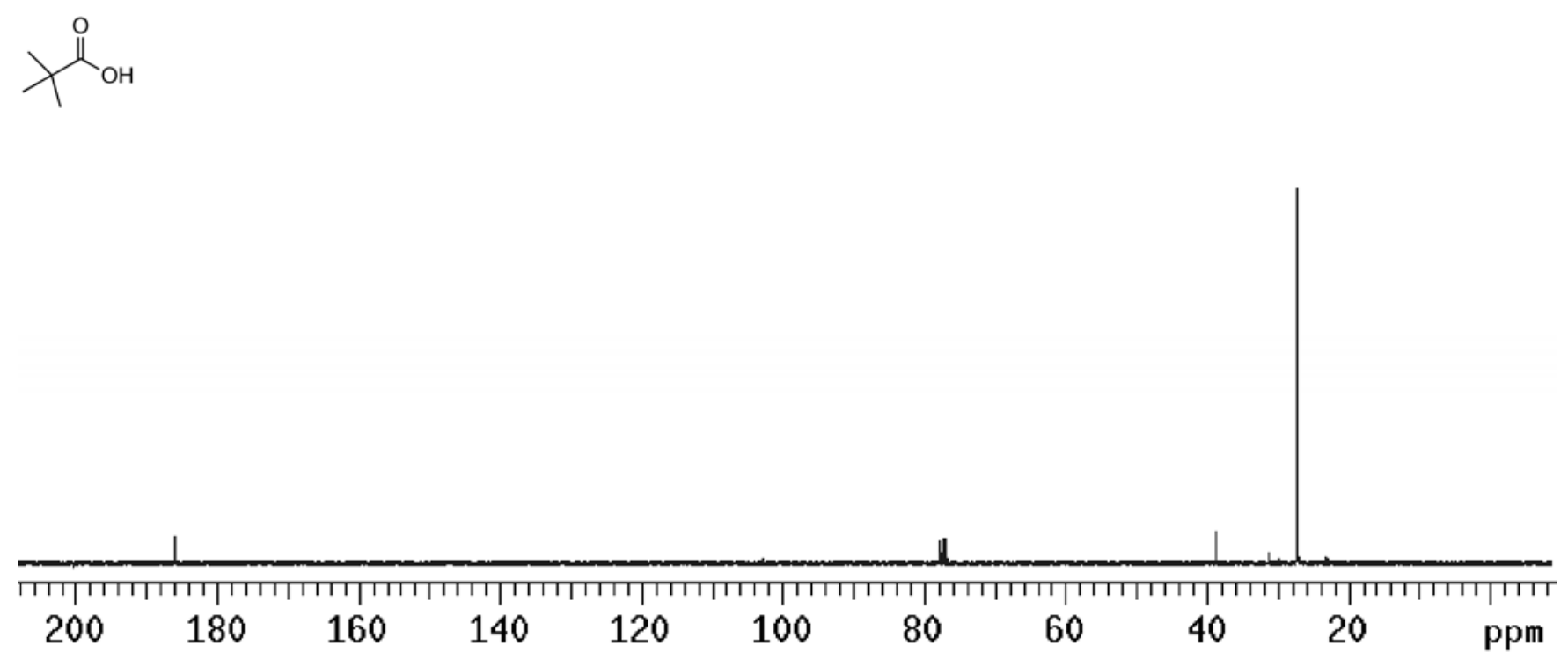
${ }^{1} \mathrm{H}$ NMR spectrum of 2-octanone. Table 2, entry 1.
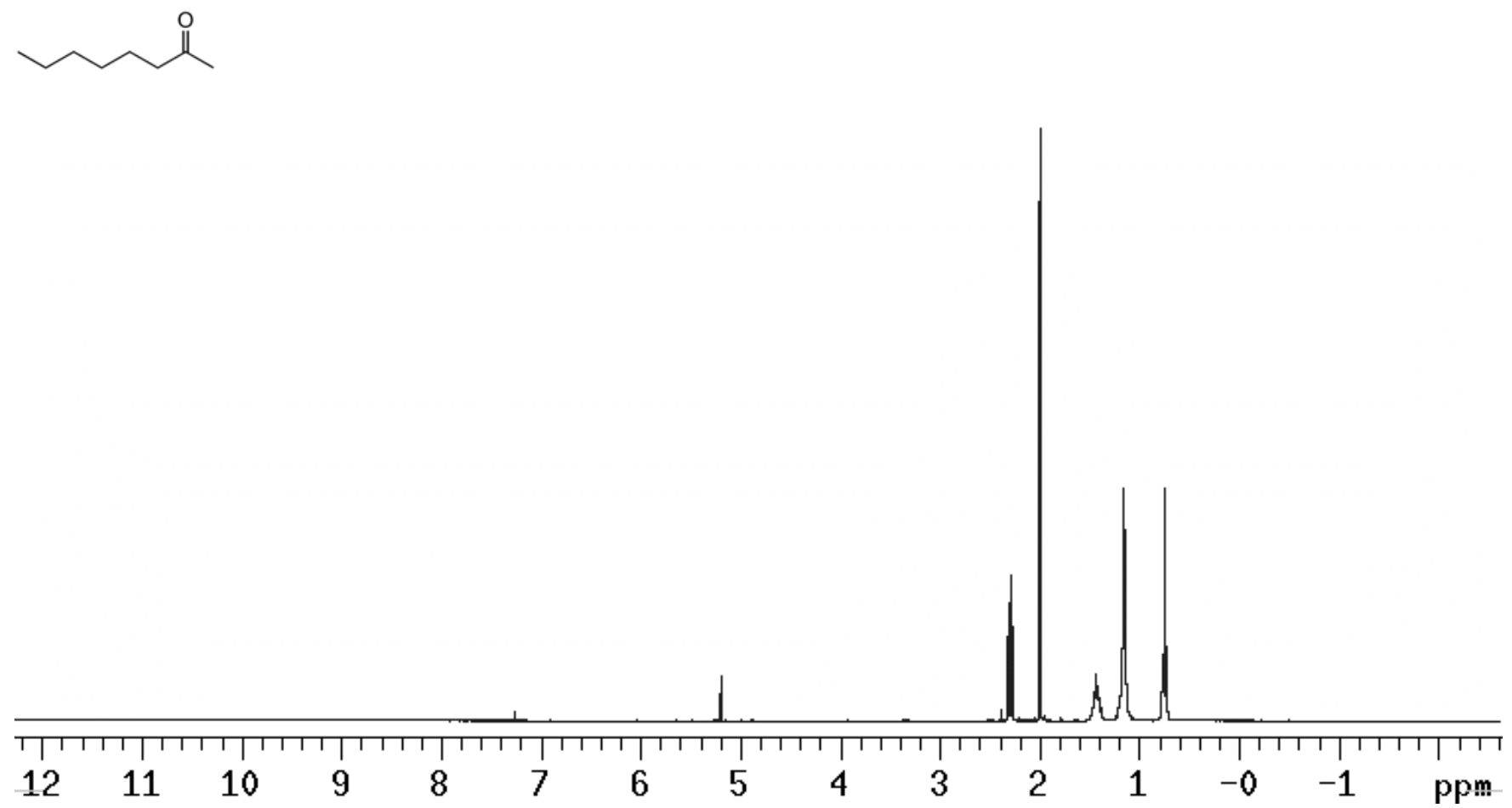

${ }^{13} \mathrm{C}$ NMR spectrum of 2-octanone. Table 2, entry 1.
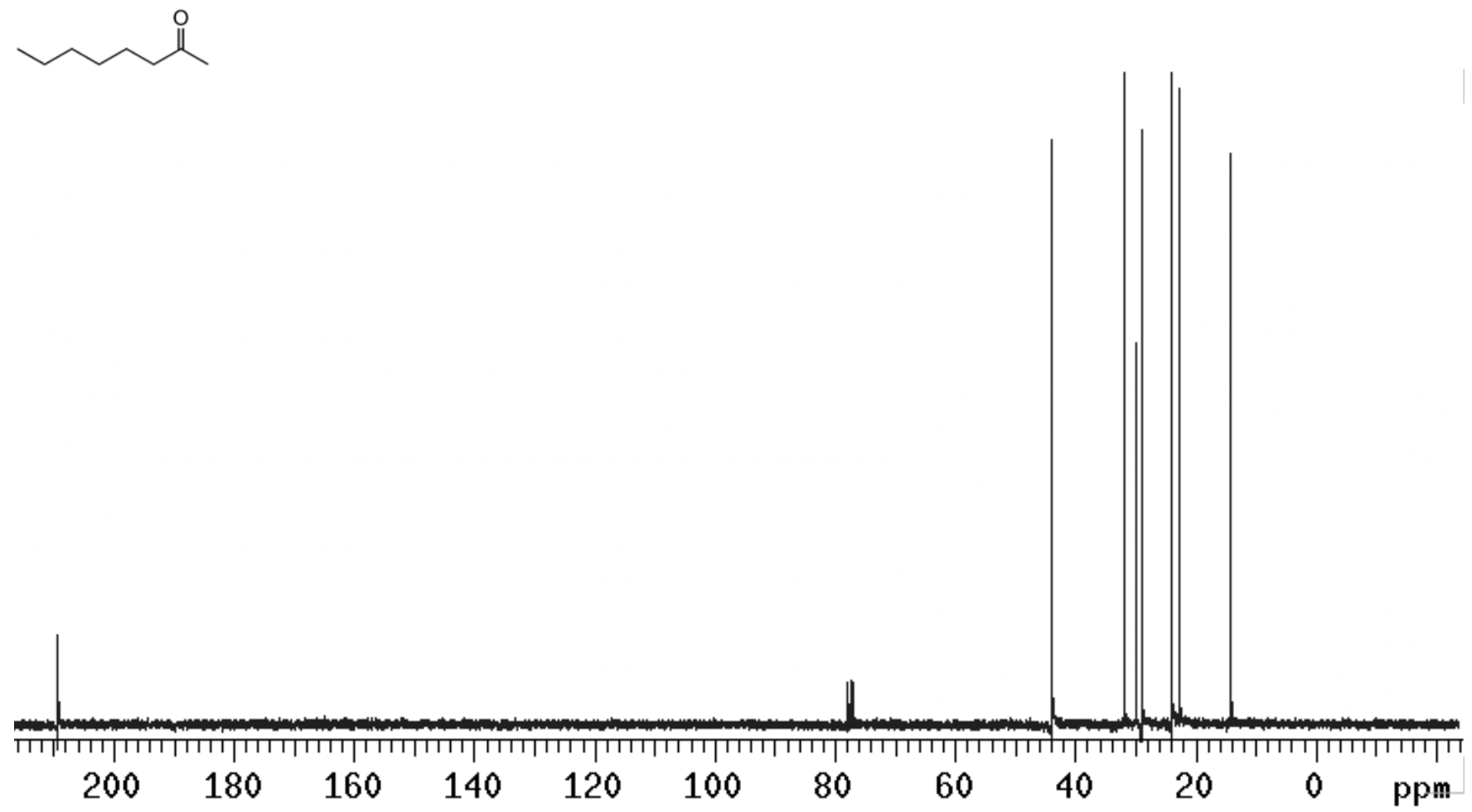
${ }^{1} \mathrm{H}$ NMR spectrum of cyclopentanone. Table 2, entry 2.<smiles>O=C1CCCC1</smiles>

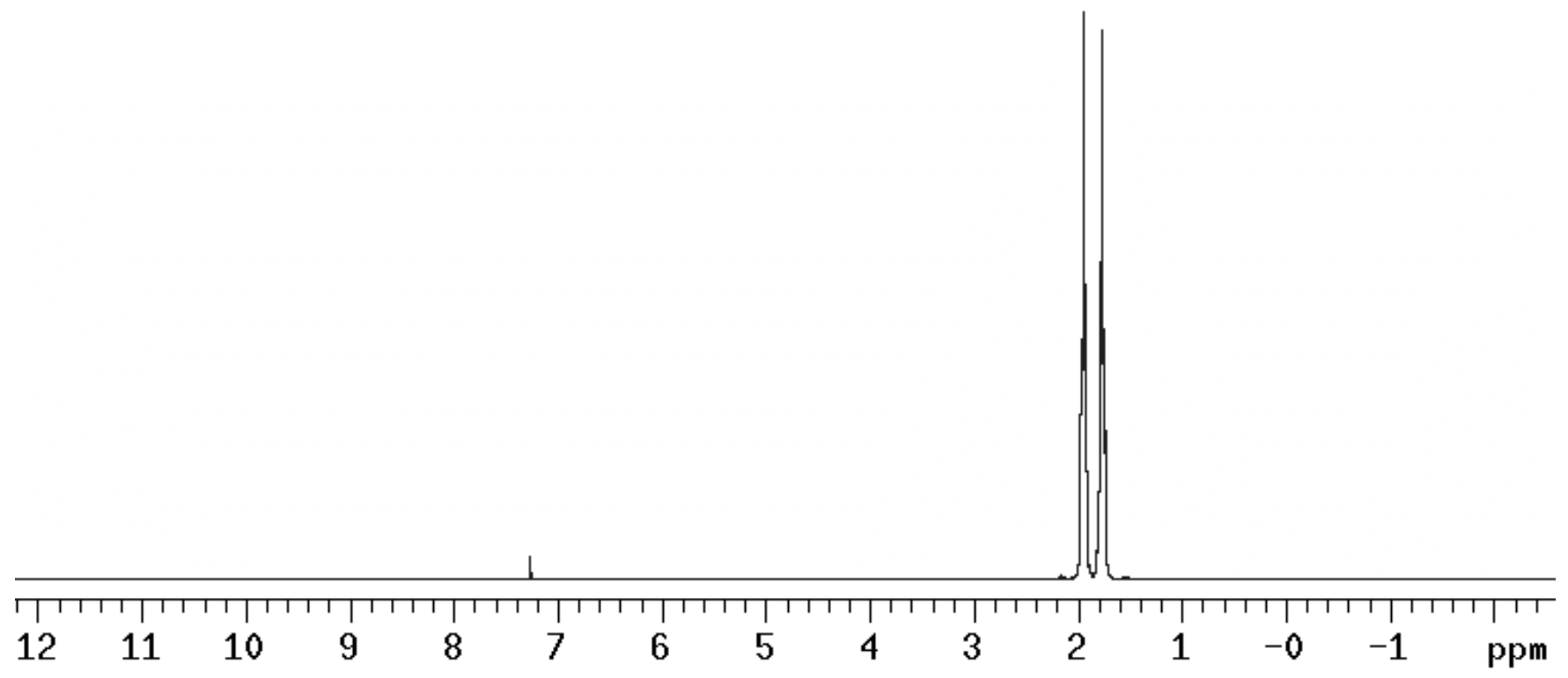

${ }^{13} \mathrm{C}$ NMR spectrum of cyclopentanone. Table 2, entry 2.
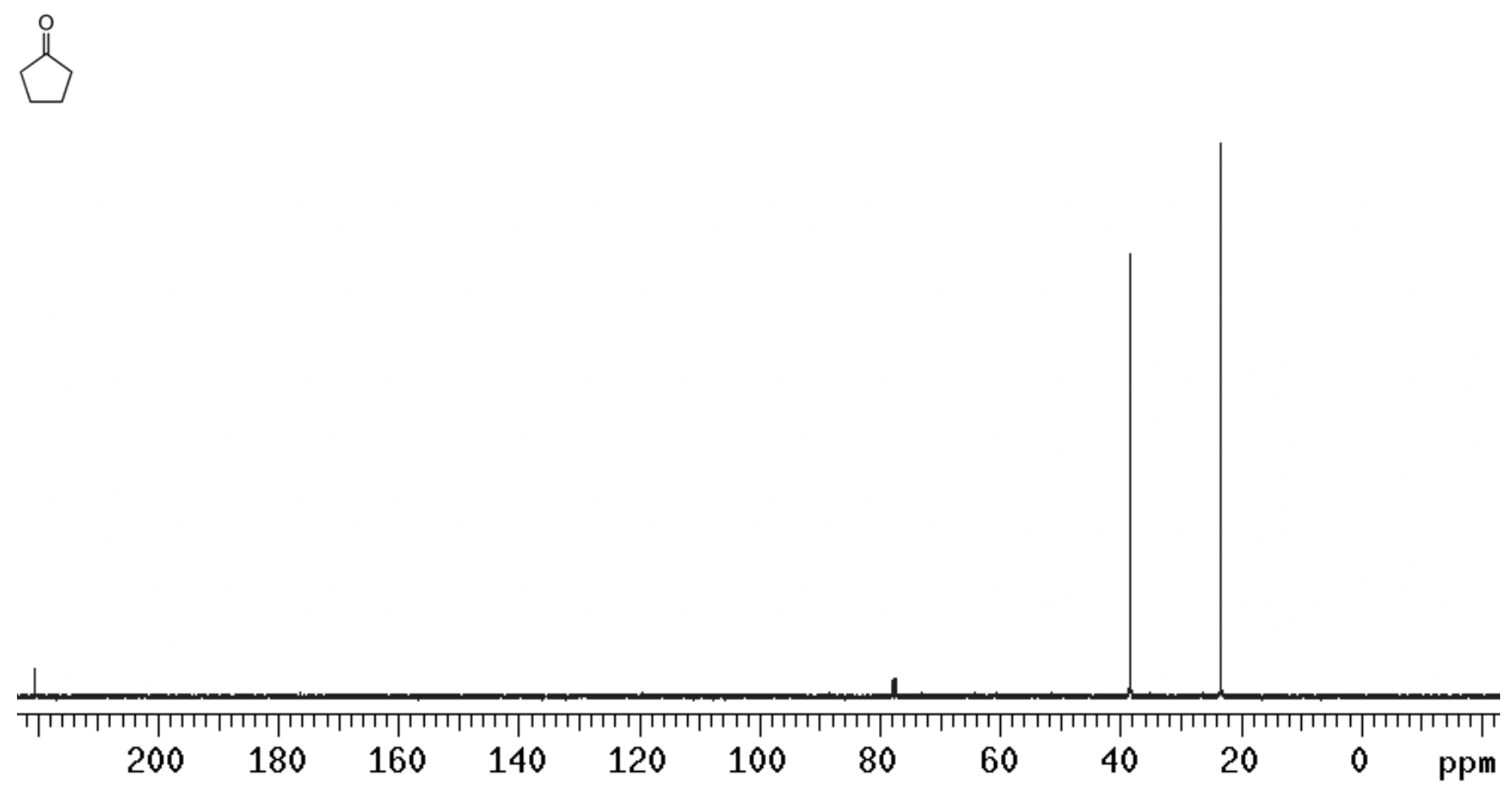
${ }^{1} \mathrm{H}$ NMR spectrum of cyclohexanone. Table 2, entry 3.

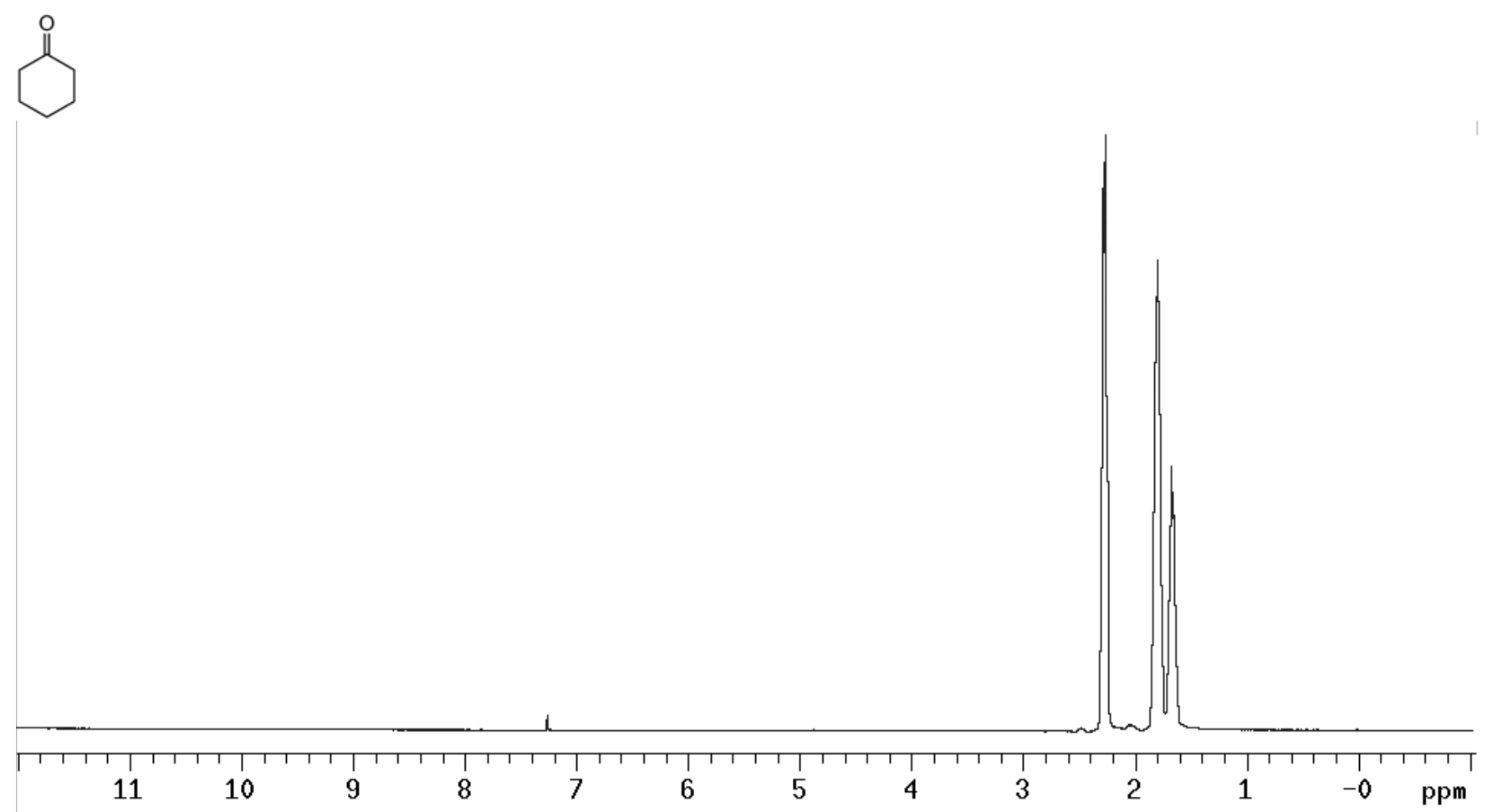

${ }^{13} \mathrm{C}$ NMR spectrum of cyclohexanone. Table 2, entry 3.
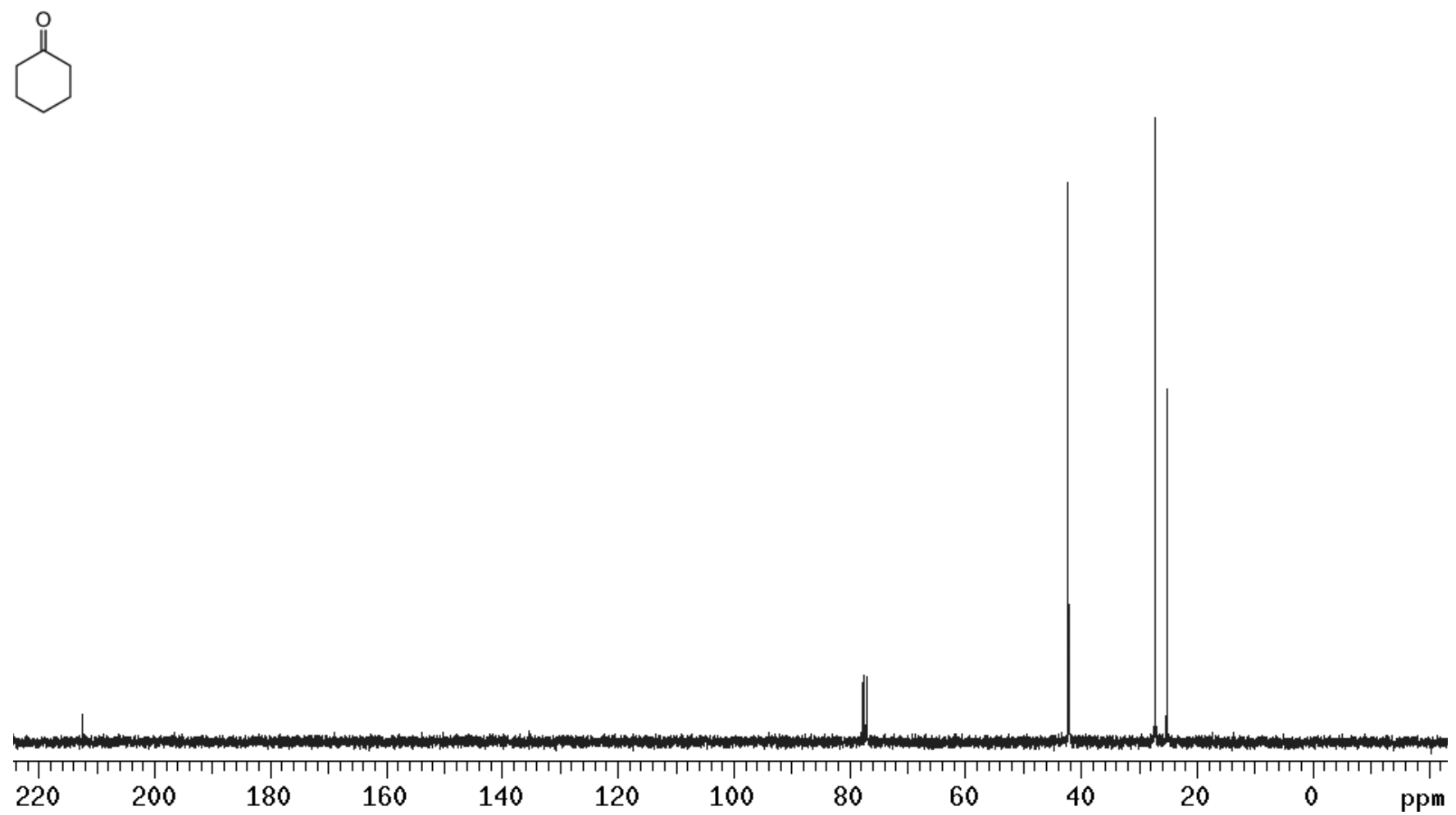
${ }^{1} \mathrm{H}$ NMR spectrum of acetophenone. Table 2, entry 4 .<smiles>CC(=O)c1ccccc1</smiles>

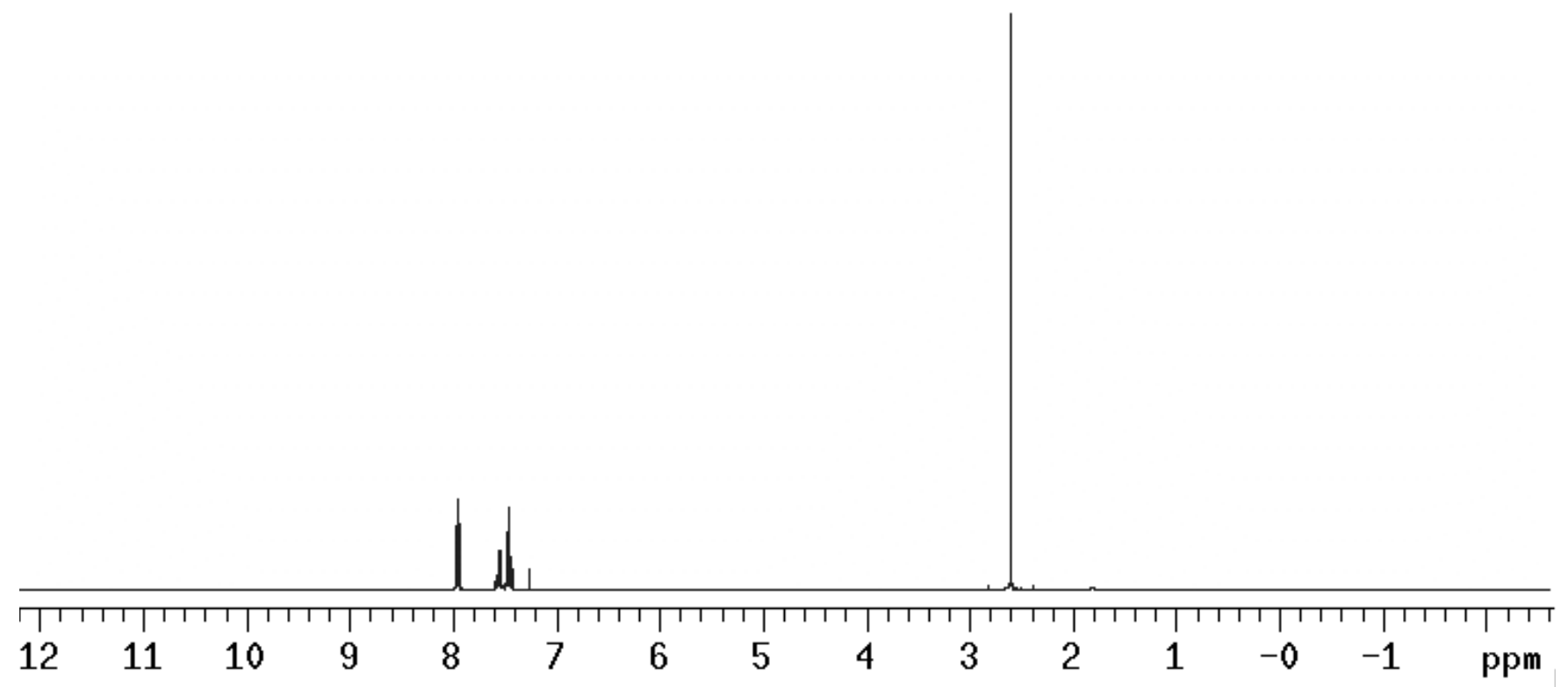

${ }^{13} \mathrm{C}$ NMR spectrum of acetophenone. Table 2, entry 4.<smiles>CC(=O)c1ccccc1</smiles>

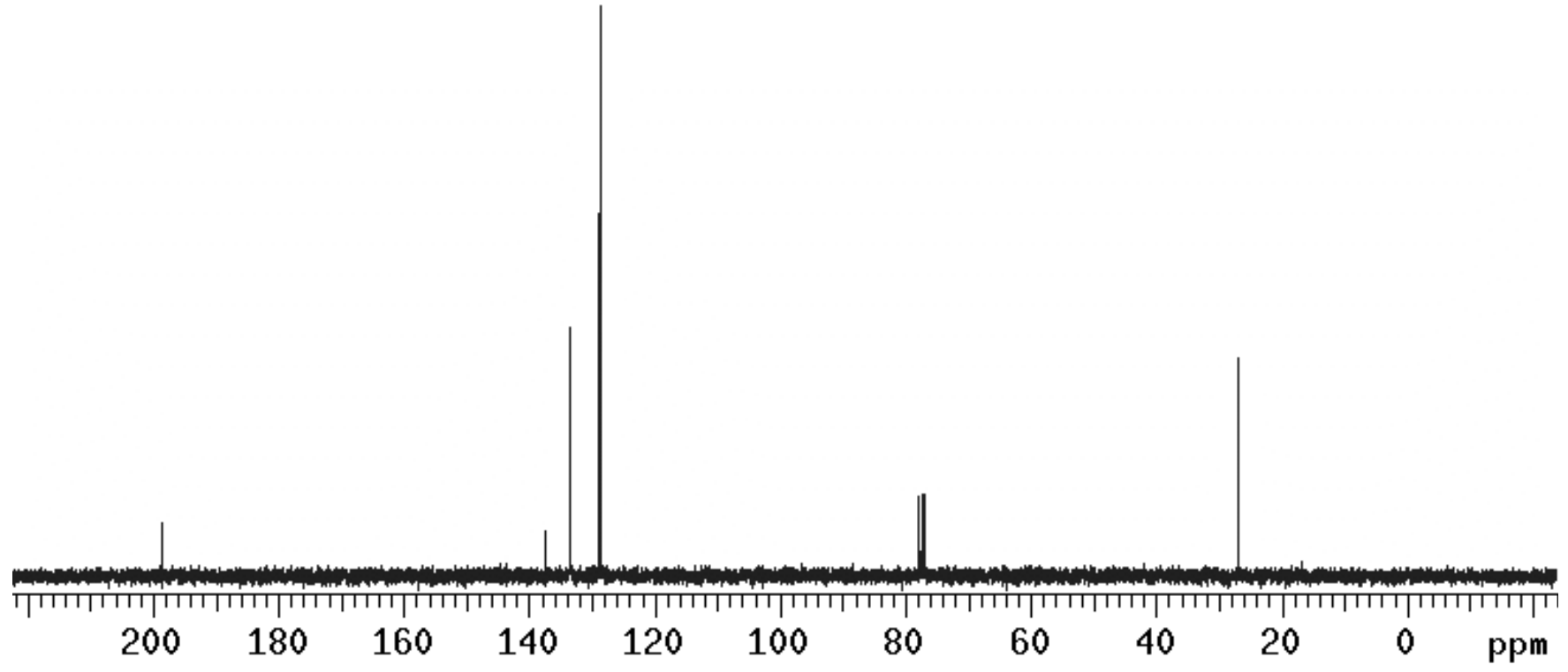


${ }^{1} \mathrm{H}$ NMR spectrum of benzophenone. Table 2, entry 5 .<smiles>O=C(c1ccccc1)c1ccccc1</smiles>

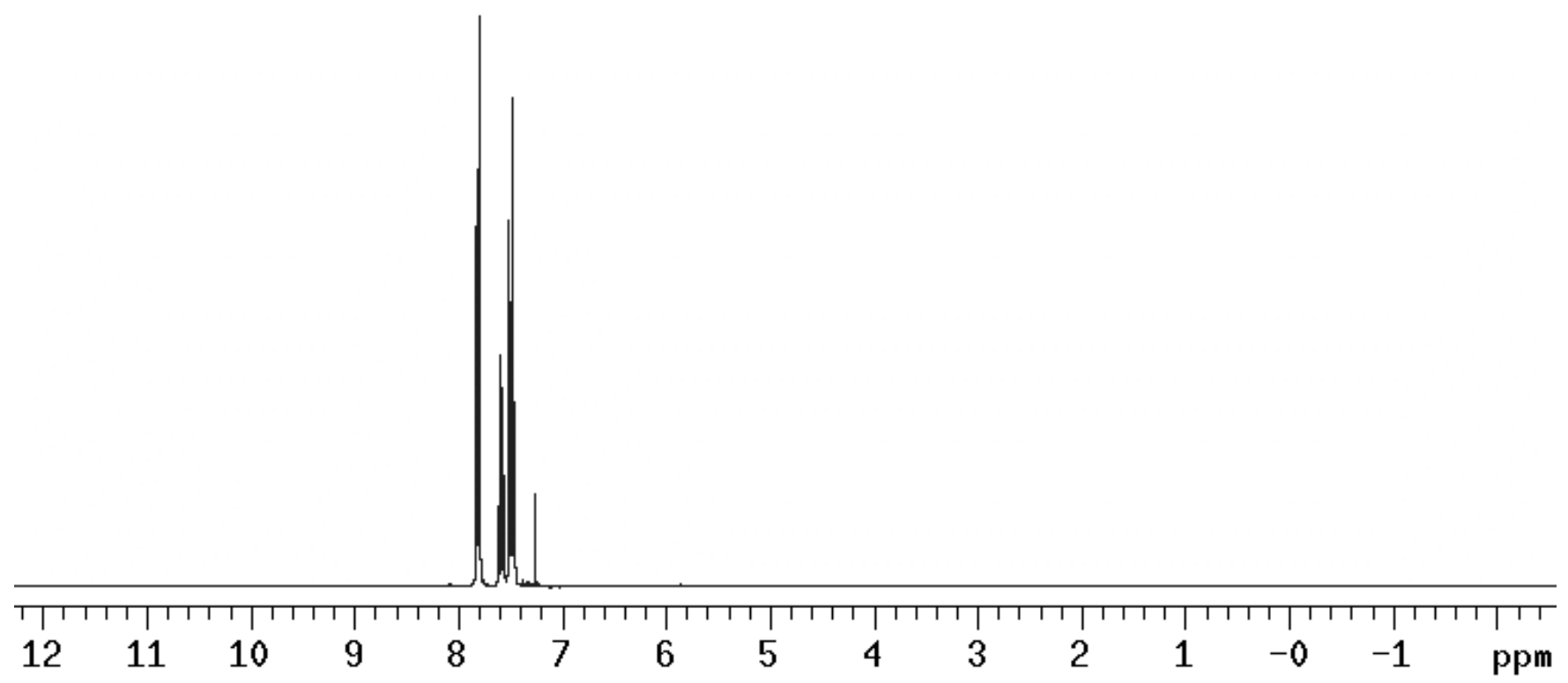

${ }^{13} \mathrm{C}$ NMR spectrum of benzophenone. Table 2, entry 5.<smiles>O=C(c1ccccc1)c1ccccc1</smiles>

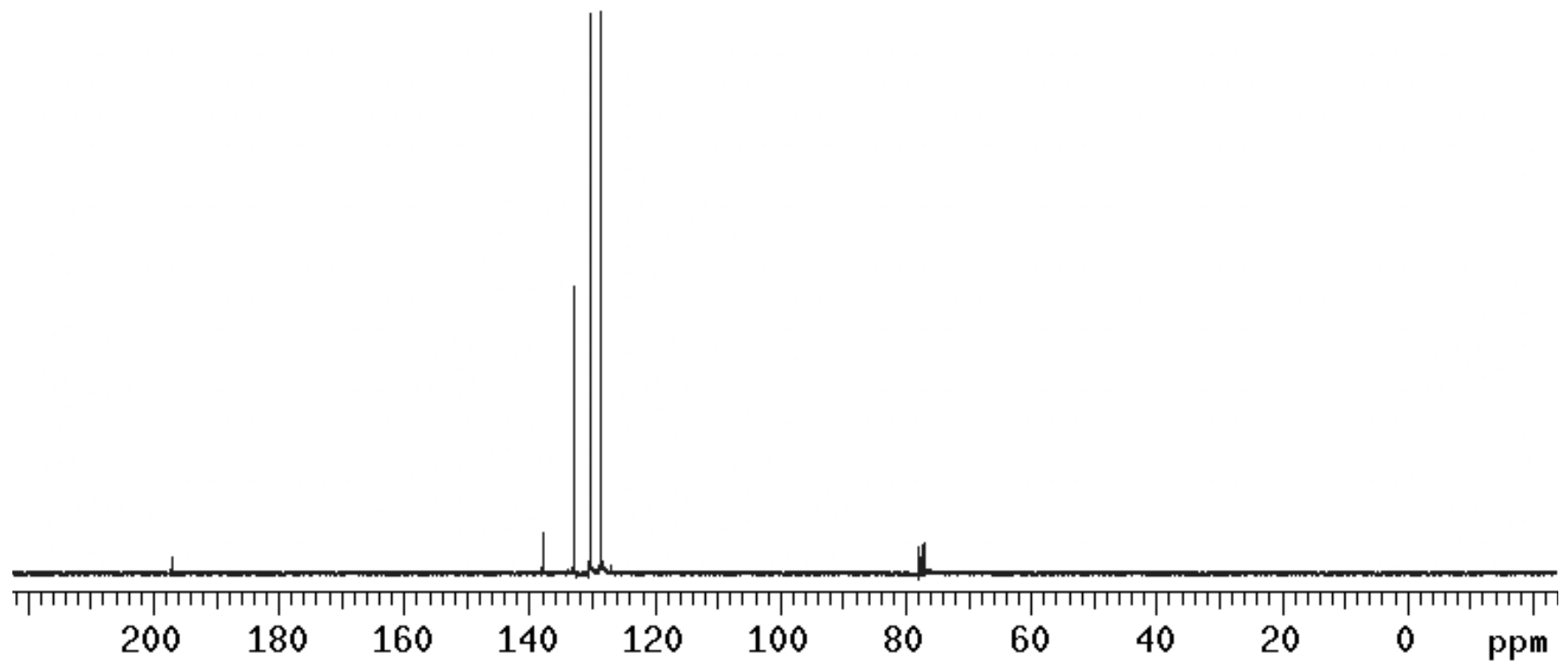


${ }^{1} \mathrm{H}$ NMR spectrum of 3-methylcyclohexanone. Table 2, entry 6.<smiles>CC1CCCC(=O)C1</smiles>

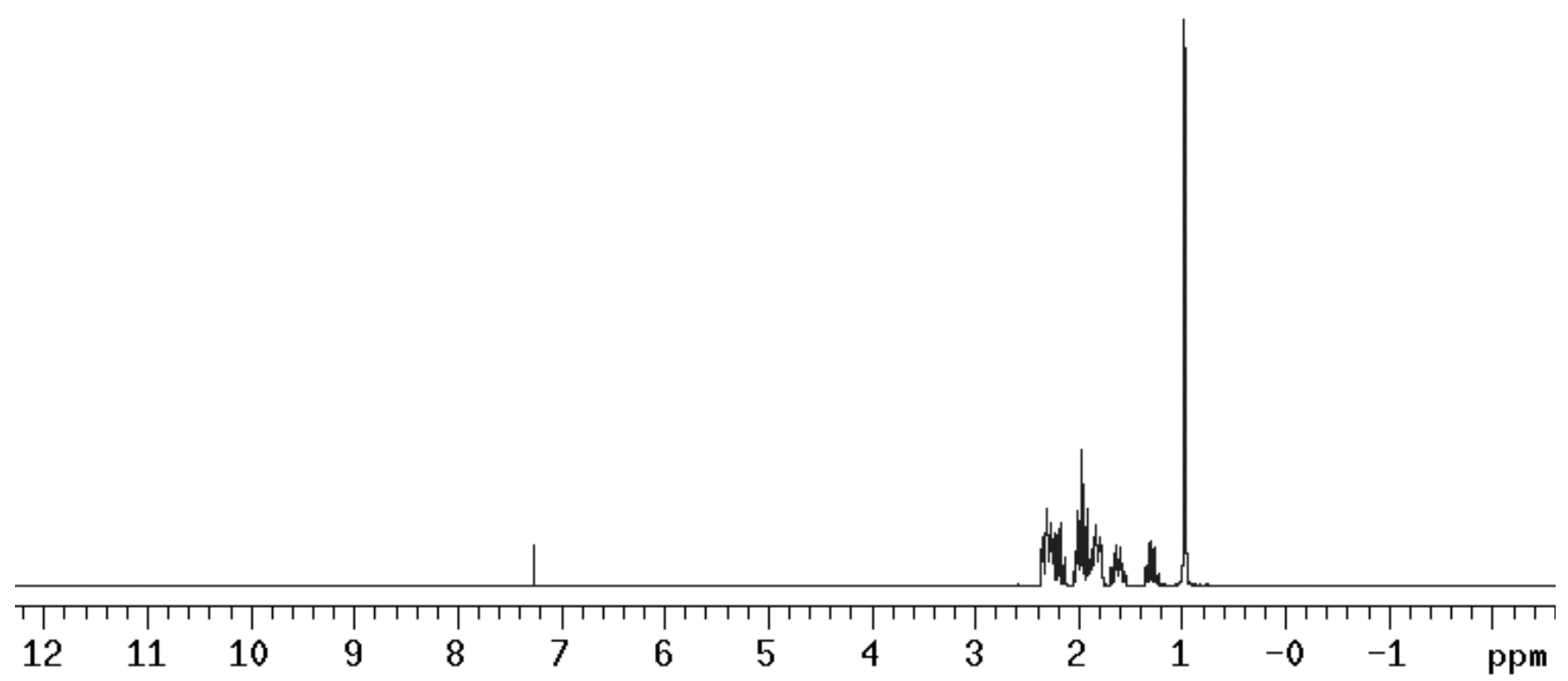

${ }^{13} \mathrm{C}$ NMR spectrum of 3-methylcyclohexanone. Table 2, entry 6.
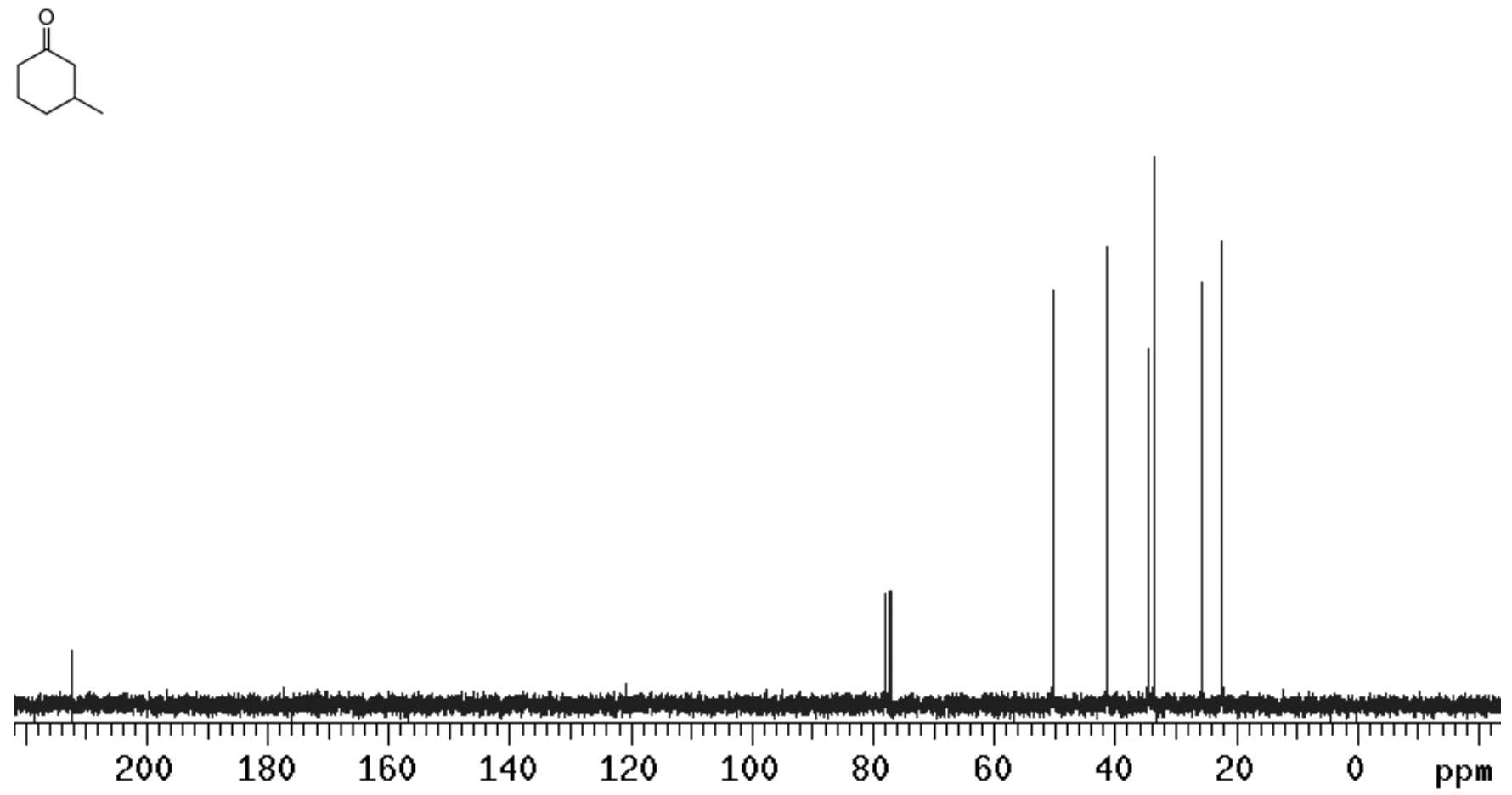
${ }^{1} \mathrm{H}$ NMR spectrum of adamantanone. Table 2, entry 7 .
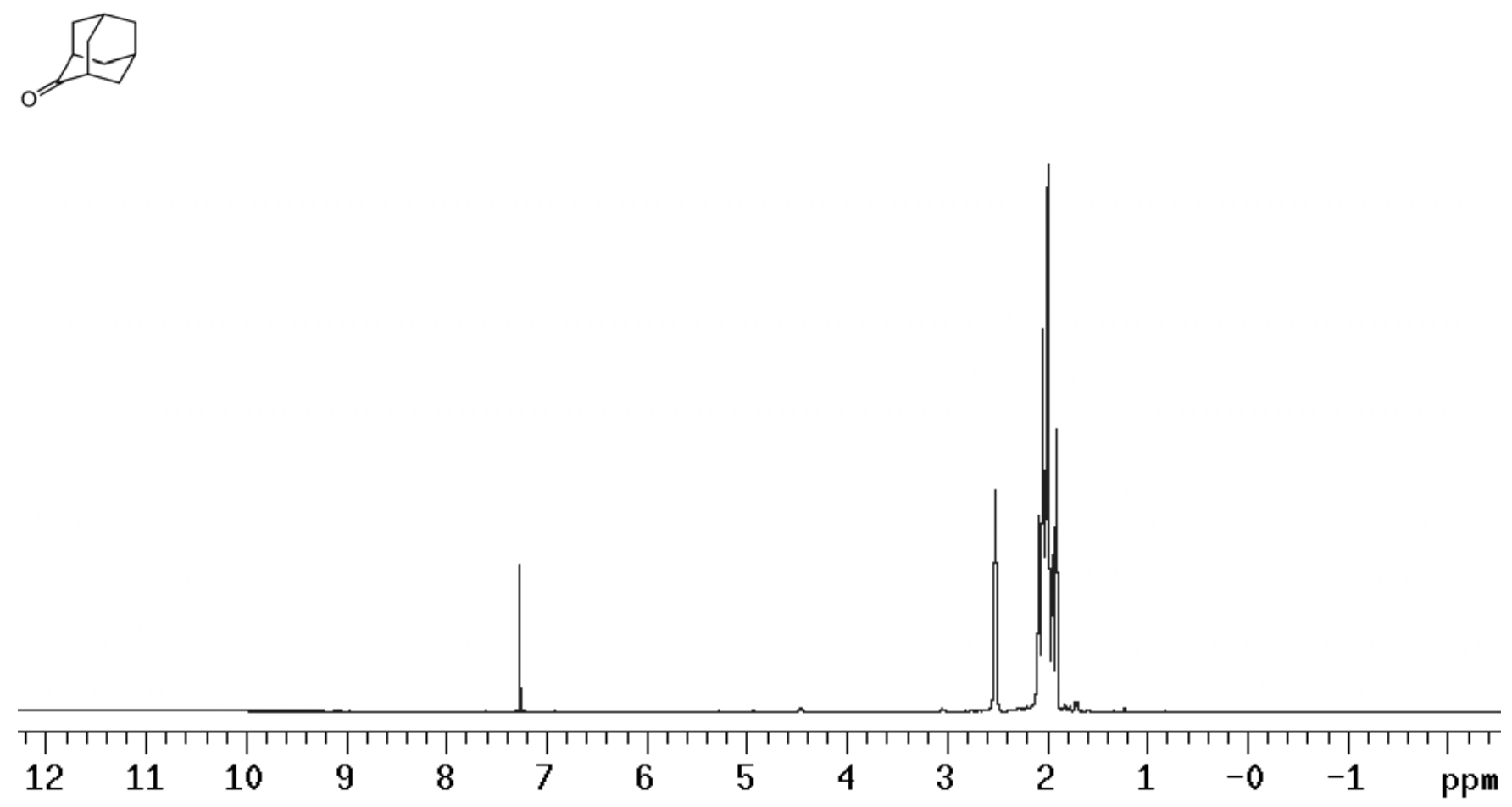

${ }^{13} \mathrm{C}$ NMR spectrum of adamantanone. Table 2 , entry 7.
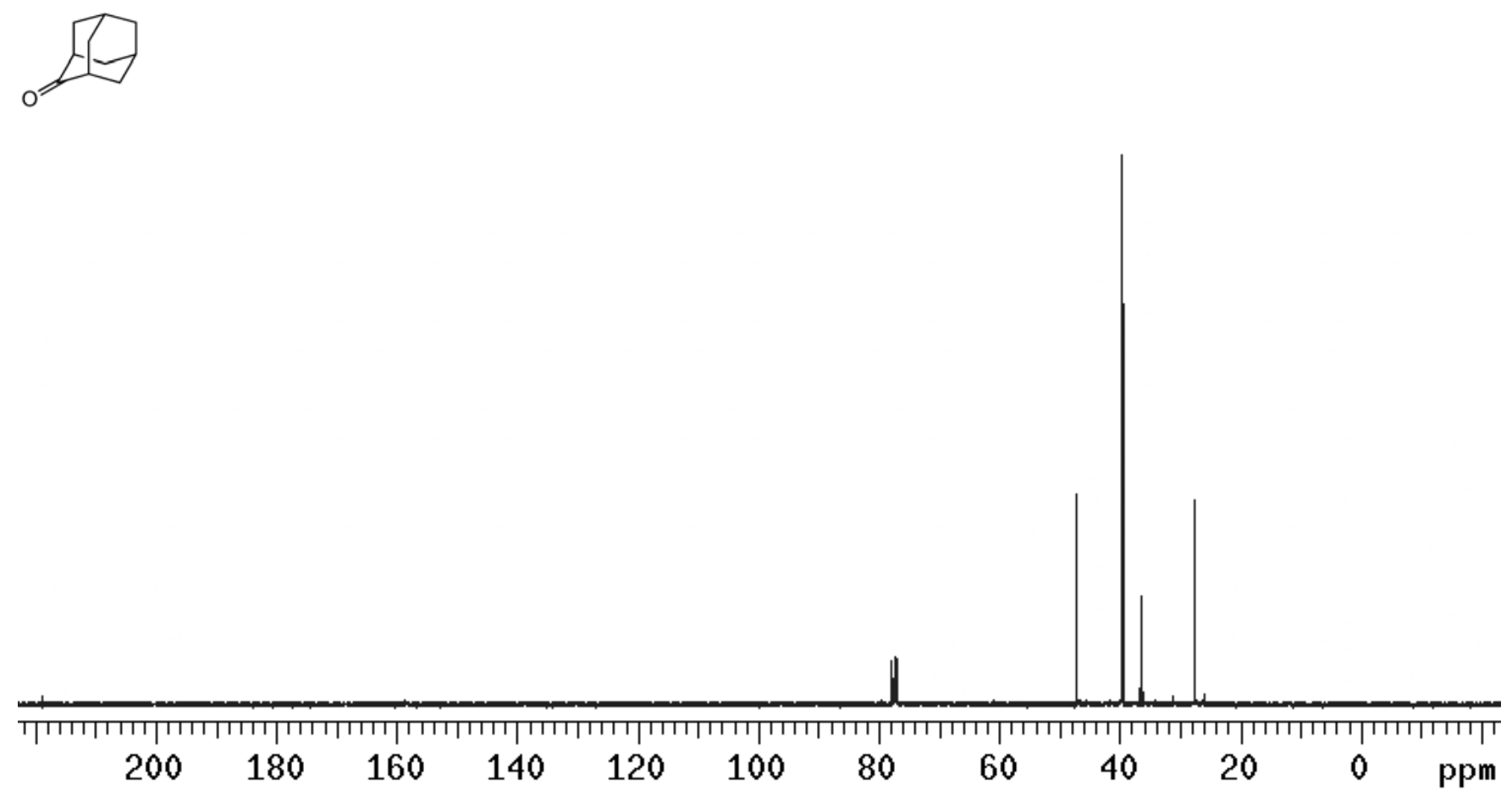
${ }^{1} \mathrm{H}$ NMR spectrum of hexanoic acid. Table 3, entry 1 .<smiles>CCCCCC(=O)O</smiles>

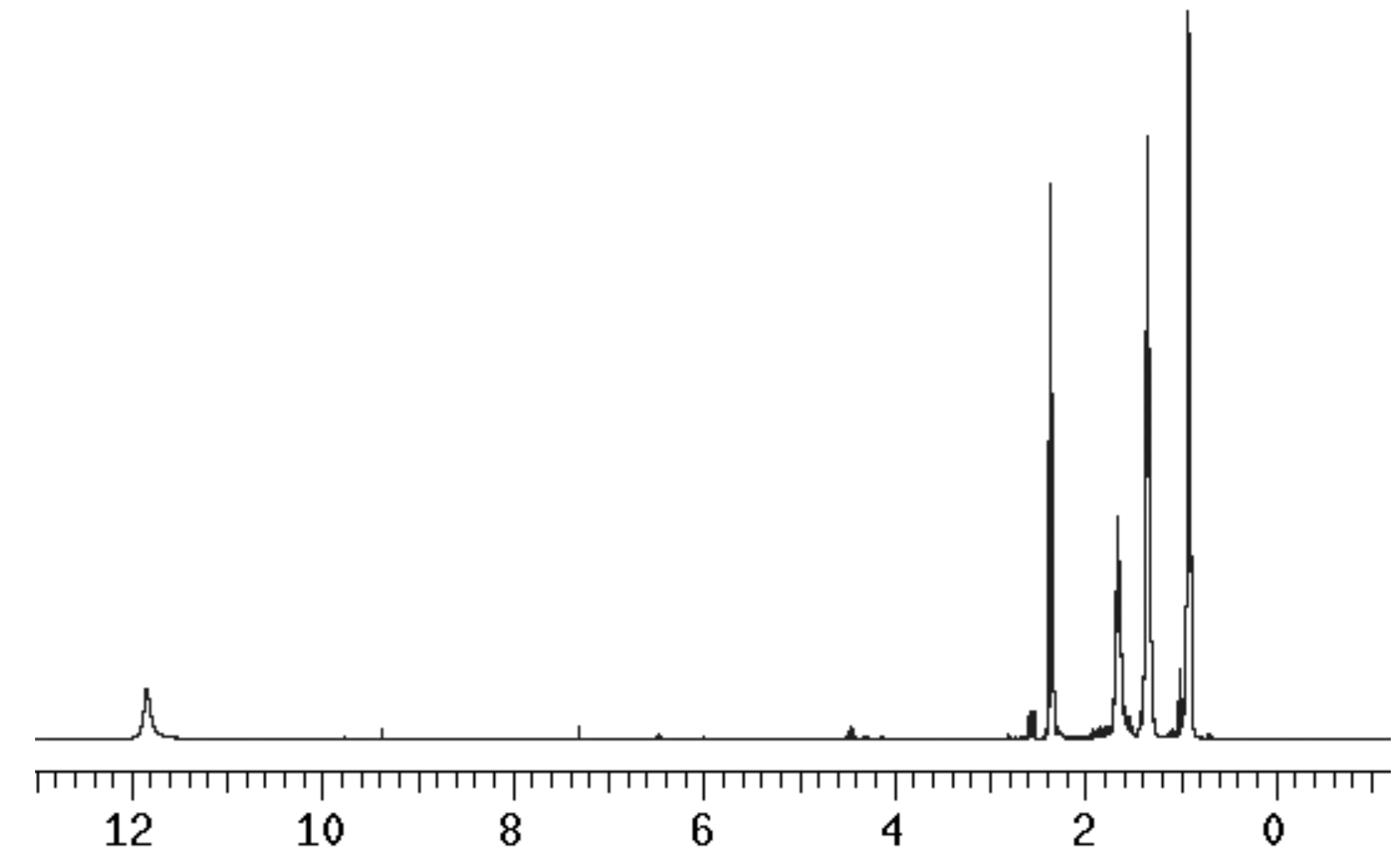

${ }^{13} \mathrm{C}$ NMR spectrum of hexanoic acid. Table 3 , entry 1.<smiles>CCCCCC(=O)O</smiles>

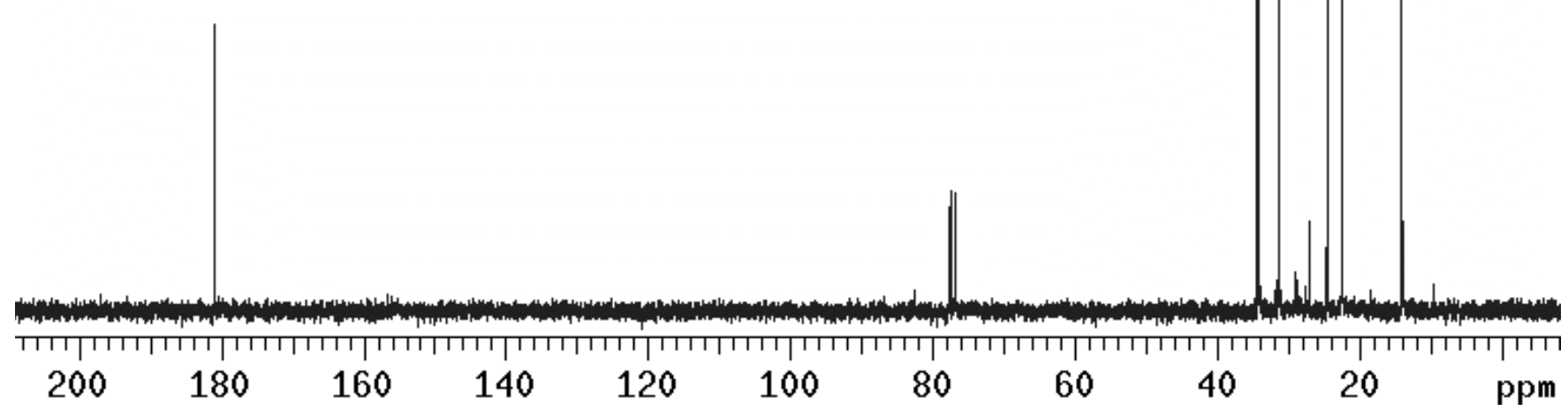


${ }^{1} \mathrm{H}$ NMR spectrum of octanoic acid. Table 3, entry 2.<smiles>CCCCCCCC(=O)O</smiles>

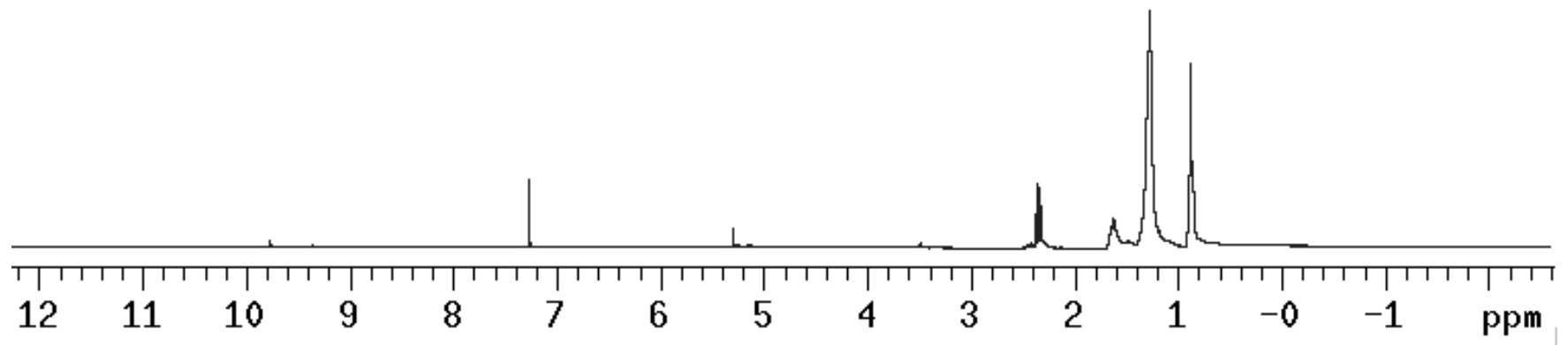

${ }^{13} \mathrm{C}$ NMR spectrum of octanoic acid. Table 3, entry 2.<smiles>CCCCCCCCC(=O)O</smiles>

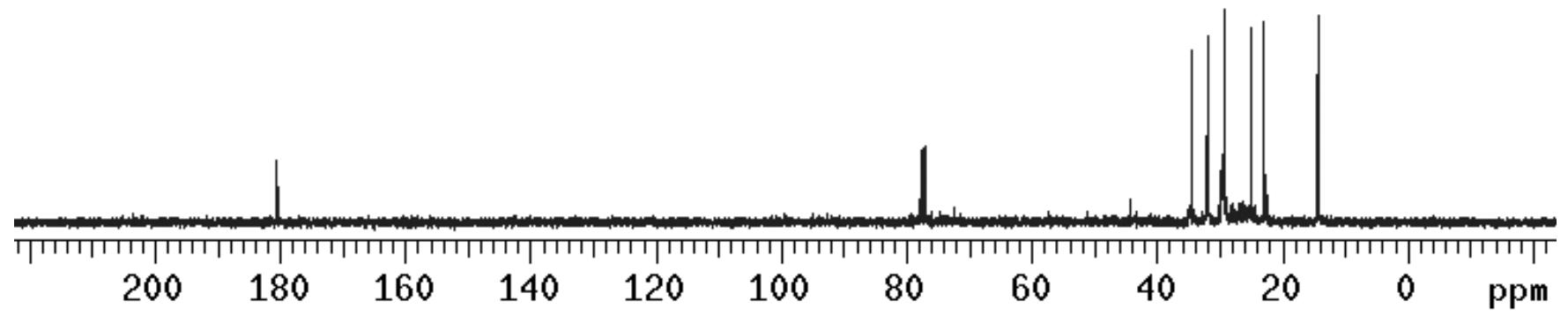


${ }^{1} \mathrm{H}$ NMR spectrum of fumaric acid.<smiles>O=C(O)/C=C/C(=O)O</smiles>

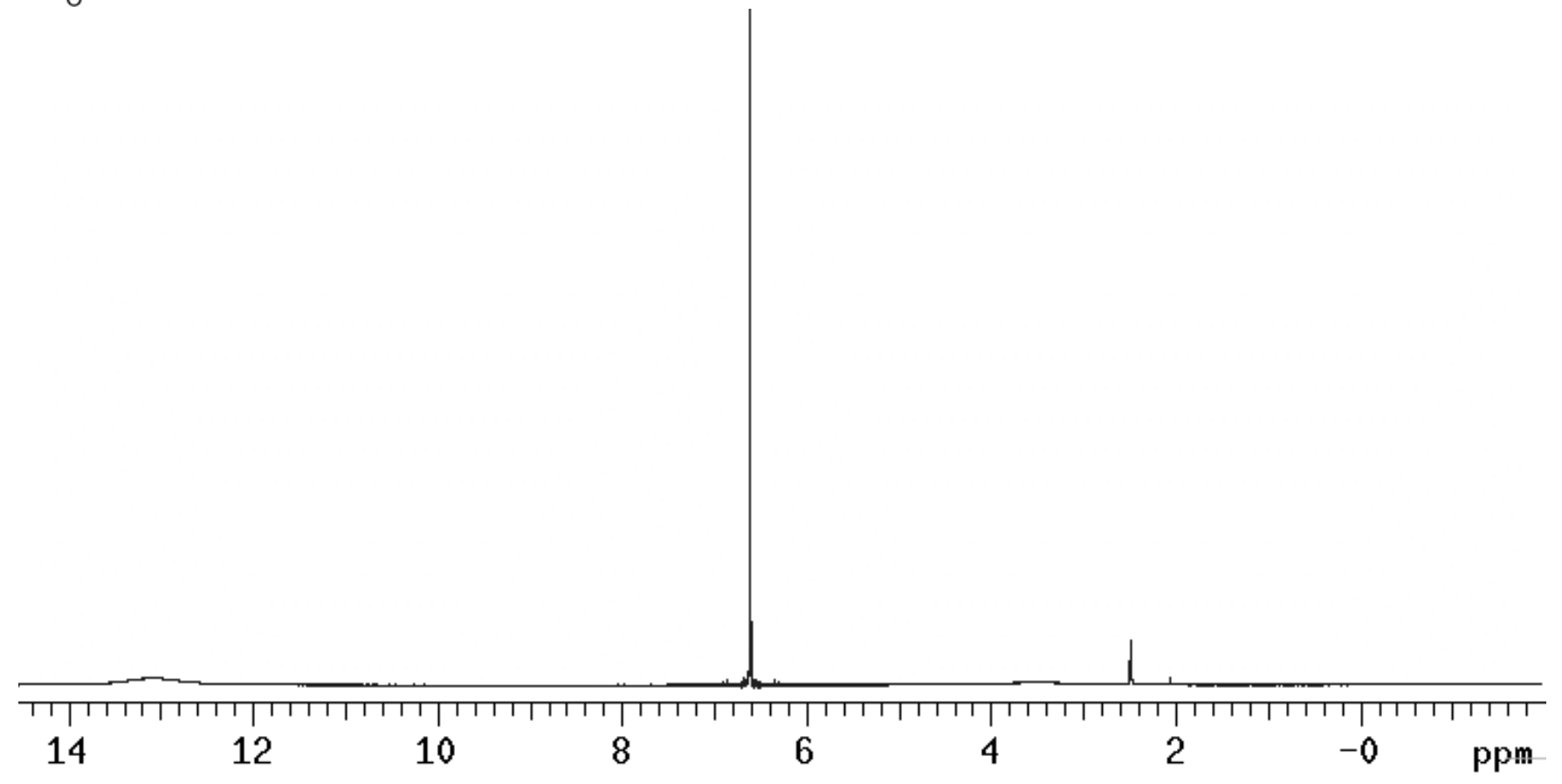

${ }^{13} \mathrm{C}$ NMR spectrum of fumaric acid.<smiles>O=C(O)C=CC(=O)O</smiles>

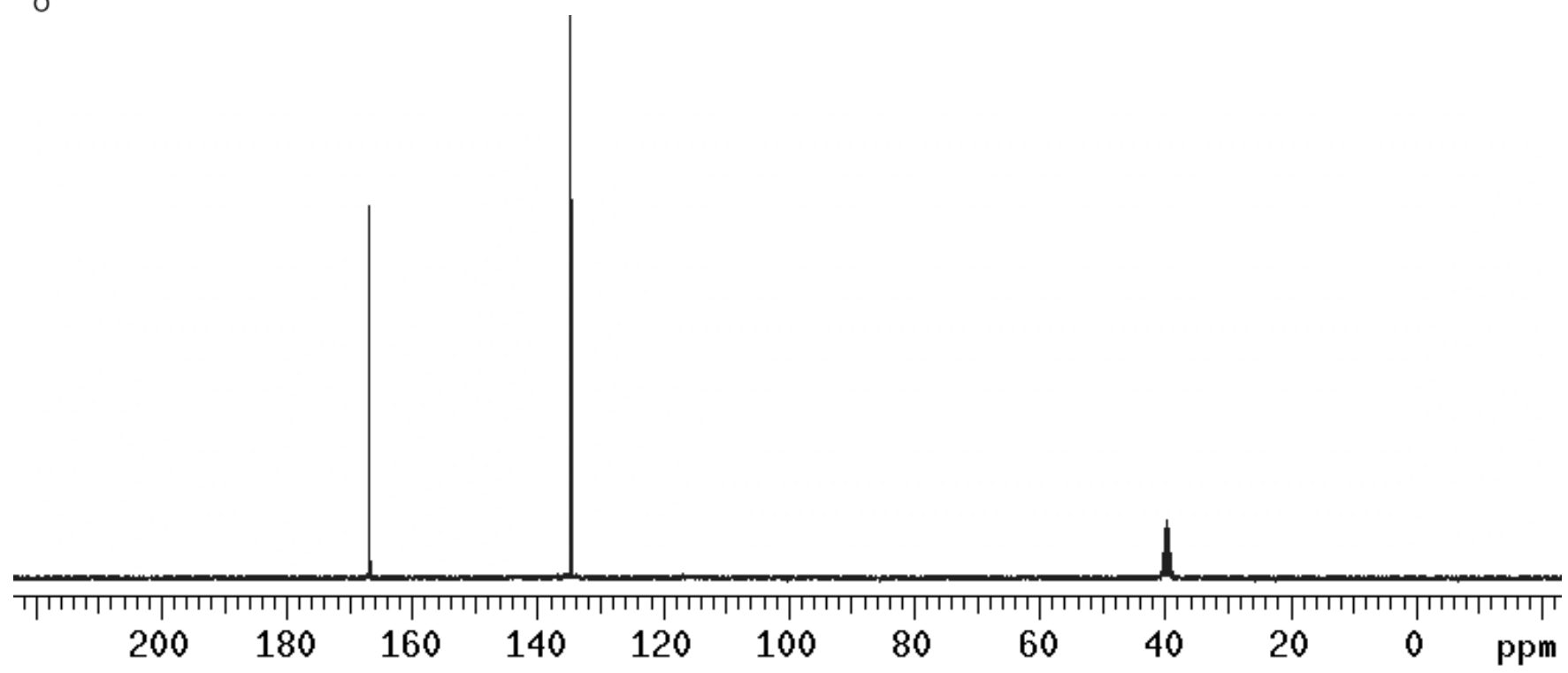


${ }^{1} \mathrm{H}$ NMR spectrum of dichloroacetic acid and monochloroacetic acid mixture (1:5).

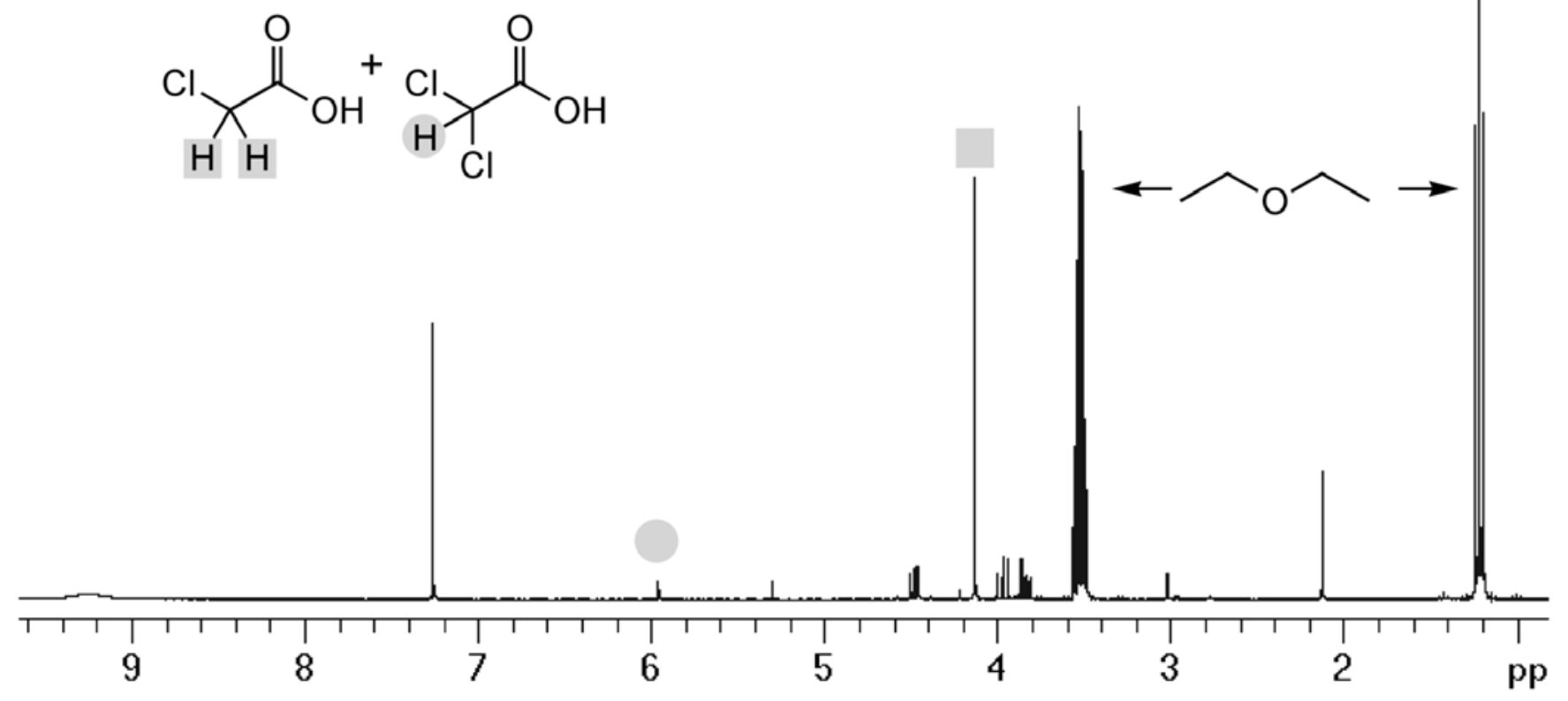


${ }^{1} \mathrm{H}$ NMR spectrum of oxiranylacetic acid.<smiles>O=C(O)CC1CO1</smiles>

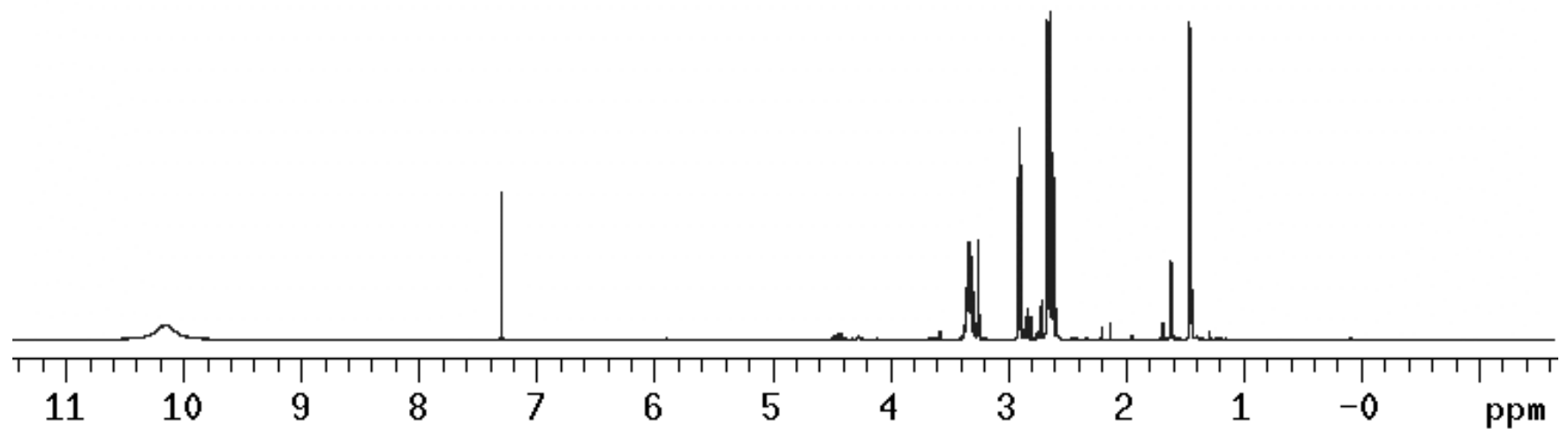

${ }^{13} \mathrm{C}$ NMR spectrum of oxiranylacetic acid.<smiles>O=C(O)CC1CO1</smiles>

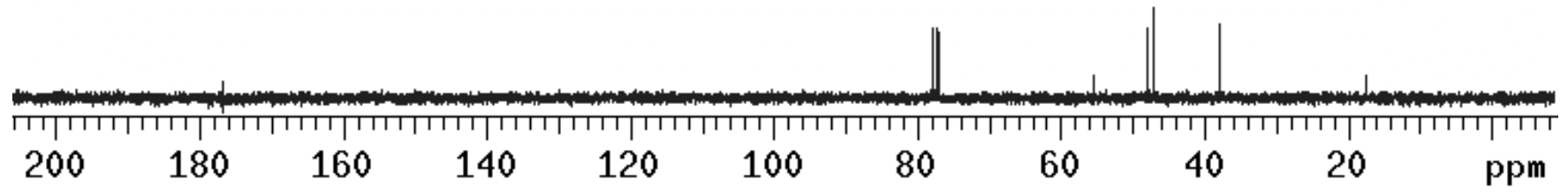

